\title{
Analysis of the spectroscopy of a hybrid system composed of a superconducting flux qubit and diamond $\mathrm{NV}^{-}$centers
}

\author{
H. Cai, ${ }^{1,2}$ Y. Matsuzaki, ${ }^{1}$ K. Kakuyanagi, ${ }^{1}$ H. Toida, ${ }^{1}$ X. Zhu, ${ }^{3}$ \\ N. Mizuochi ${ }^{4}$ K. Nemoto,${ }^{5}$ K. Semba $^{6}$ W. J. Munro, ${ }^{1}$ S. Saito, ${ }^{1}$ \\ H. Yamaguchi, ${ }^{1,2}$ \\ ${ }^{1}$ NTT Basic Research Laboratories, NTT Corporation, Atsugi, Kanagawa, 243-0198, \\ Japan \\ ${ }^{2}$ Department of Physics, Tohoku University, Sendai, Miyagi 980-8578, Japan \\ ${ }^{3}$ Institute of Physics, Chinese Academy of Sciences, Beijing, 100190, China \\ ${ }^{4}$ Graduate School of Engineering Science, University of Osaka, 1-3 Machikane-yama, \\ Toyonaka, Osaka, 560-8531, Japan \\ ${ }^{5}$ National Institute of Informatics, 2-1-2 Hitotsubashi, Chiyoda-ku, Tokyo, 101-8430, \\ Japan \\ ${ }^{6}$ National Institute of Information and Communications Technology, 4-2-1, \\ Nukuikitamachi, Koganei-city, Tokyo, 184-8795, Japan. \\ E-mail: matsuzaki.yuichiro@lab.ntt.co.jp
}

\begin{abstract}
.
A hybrid system that combines the advantages of a superconductor flux qubit and an electron spin ensemble in diamond is one of the promising devices to realize quantum information processing. Exploring the properties of the superconductor diamond system is essential for the efficient use of this device. When we perform spectroscopy of this system, significant power broadening is observed. However, previous models to describe this system are known to be applicable only when the power broadening is negligible. Here, we construct a new approach to analyze this system with strong driving, and succeed to reproduce the spectrum with the power broadening. Our results provide an efficient way to analyze this hybrid system.
\end{abstract}




\section{Introduction}

The properties of different types of qubits have been studied extensively over the past decade toward realizing quantum computation. Each system has its own advantages and disadvantages. The idea of combining different systems is pursued by several groups, which would inherit the advantages of each system, such as atoms coupled to optical cavities, spins coupled to resonators, and spins coupled to superconducting qubits [1, 2, 3, 4, 6, 7, 8].

One of the most successful hybrid schemes is to couple the electron spin ensemble with superconducting circuit to realize the fast processing and long storage times. Although an electron spin ensemble may possess excellent coherence properties, the weak non-linearity makes it difficult to realize a large scale quantum computer. Superconducting qubits are sensitive to the noise which leads to a relatively short coherence time. Instead, due to a nonlinearity that Josephson Junctions has, a superconducting circuit has excellent controllability of quantum states involved in the processing, such as single qubit gates, two qubit gates, and projective measurements [9, 10, 11, 12, 13, 14, 15, 16, 17. There are two major schemes that utilize this type of hybrid system. One of them consists of a spin ensemble, a superconducting resonator, and a superconducting transmon qubit [18, 19, 20, 21, 22]. Here, the resonator plays the role of a quantum bus [8, 14] where the resonator mediates the interaction between the spins and a superconducting qubit. The other one is to use a direct coupling between a spin ensemble and the superconducting flux qubit (FQ) [7, 23, 24, 25, 26].

A particularly attractive spin ensemble for such hybrid systems is negatively charged nitrogen vacancy centers $\left(\mathrm{NV}^{-}\right)$in the diamond crystal whose coherence time is around $0.6 s$ [27, 28]. An $\mathrm{NV}^{-}$center is a defect consisting of a substitutional nitrogen atom and an adjacent vacancy where an additional electron is trapped. It is known that an $\mathrm{NV}^{-}$center is a spin- 1 system, and there is an energy splitting of $2.88 \mathrm{GHz}$ between the states $|0\rangle$ and $| \pm 1\rangle$ without an applied magnetic fields. Furthermore, the $\mathrm{NV}^{-}$centers can be strongly coupled with superconducting circuits by a super-radiant effect [18, 19], where the coupling strength is enhanced by a factor of $\sqrt{N}$.

Several groups have already demonstrated the hybrid coupling systems by the ensemble of electron spins and superconducting circuit resonator [20, 21, 22, 29, 30, 31, 32]. Also, Further, Kubo et al. [20] has succeeded in indirectly coupling the ensemble to the transmon qubit using a frequency tunable resonator to mediate it. However, in order to enhance the coupling strength, this system requires a large number of electron spins $\left(\sim 10^{12}\right)$ in the ensemble to achieve the coupling strength of tens of $\mathrm{MHz}$ [20, 21, 22, 29, 30, 31, 32. These experiments need the mm-size resonator, which could be difficult for the integration of many memories on a single quantum based chip [21, 22].

The advantage to use the spin ensemble and the superconducting flux qubit [7, 23, 24] is that the superconducting flux qubit can couple directly and more strongly with the spin ensemble due to the persistent current of the flux qubit around $300 \mathrm{nA}$ 
$\sim 900 n A$ [33, 34]. This system requires a much smaller number of $\mathrm{NV}^{-}$centers spins $\left(\sim 10^{7}\right)$ to realize a strong coupling regime, and the sample occupies only a surface area of tens of micro-meter square. Coherent strong coupling between a flux qubit and nitrogen-vacancy $\left(\mathrm{NV}^{-}\right)$centers has already been demonstrated [23, 24, 25], as well as the coherent exchange of a single quantum excitation [34. Quantum memory operations involving single qubit and entangled states have been implemented [24].

Some theoretical models have been investigated to describe such hybrid systems [7, 18, 19, 35]. Traditionally, Jaynes-Cummings model (JC model) is used to describe the coupling between the flux qubit and the spin ensemble [7, 18, 19, 35]. Under a strong external magnetic field, the degenerate state $|1\rangle$ and $|-1\rangle$ of the $\mathrm{NV}^{-}$center would be separated far from each other and the $\mathrm{NV}^{-}$center can be considered as a spin $1 / 2$ system (two-level system). If the number of excitations in the hybrid system is much smaller than the number of $\mathrm{NV}^{-}$centers, we can treat this system as a harmonic oscillator and then use JC model to describe the coupling between the flux qubit and $\mathrm{NV}^{-}$centers. However, when the applied magnetic field is weak, $\mathrm{NV}^{-}$center will show the properties of a spin 1 system (three-level system) 25 because of the degenerate states of $|1\rangle$ and $|-1\rangle$. Therefore, the conventional model cannot be straight-forwardly applied to analyze the properties of flux qubit coupled with $\mathrm{NV}^{-}$center for this regime.

From the spectroscopic measurement in a superconductor diamond system without an applied magnetic field, three resonant peaks have been observed in [21, 34]. A sharp peak has been clearly observed in the middle of the avoided crossing caused by the coherent coupling between the superconducting circuits and an ensemble of electron spins in nitrogen-vacancy center. The center narrow peak shows a longer lifetime than that of the other two broader peaks, which may provide an alternative approach for the quantum memory [25, 36.

In the paper [25], a full Hamiltonian model for the hybrid system of the flux qubit and $\mathrm{NV}^{-}$centers is used to interpret the mechanism causing the sharp narrow peak, which contains the properties of spin 1 system such as inhomogeneous strain, randomized magnetic field from P1 center (substitutional nitrogen centers in diamond), and zero field splitting fluctuation [25]. The $\mathrm{NV}^{-}$center is regarded as a three-level system, and it was shown that two collective modes of the $\mathrm{NV}^{-}$ensembles are relevant for the spectrum. The two broader peaks are associated with the dressed bright state in $\mathrm{NV}^{-}$that can be directly coupled with the flux qubit. The center narrow peak arises from another specific collective mode that cannot be directly driven by the flux qubit. This is called a "dark state". Due to the effects of the strain and Zeeman splitting, the bright state and dark state can exchange the excitation, while the bright states interact with the flux qubit. In that theoretical analysis [25, the flux qubit is regarded as a harmonic oscillator in the limit of low excitation energy so that the Heisenberg equations can be solved in the frequency domain. However, this approximation is valid only when the driving power is weak, and will be invalid to describe the phenomenon when the power broadening is observed in the strong driving power case. Since it is not always possible to obtain a reliable data without power broadening due to a small signal to noise ratio, 
this could be a limitation to describe the hybrid system for such a regime. Therefore, it is necessary to build an alternative approach that can include the effect of power broadening in the strong driving regime.

In this paper, we extend the previous approach to reproduce the experimental spectroscopy that is significantly affected by the power broadening. The rest of this paper is organized as follows. In section 2, we introduce the previous model for this hybrid system [25]. In section 3, we highlight a new theoretical model to show that the numerical results agree with the experiments. Finally, section 4 contains a summary of our results.

\section{Hamiltonian}

Let us review the Hamiltonian introduced by the paper in [25]. The diamond containing $\mathrm{NV}^{-}$centers is attached on top of the flux qubit so that these two system can interact each other, as described in the Appendix 5.1. The Hamiltonian of this system includes not only the interaction between the flux qubit and $\mathrm{NV}^{-}$centers, but also the strain distributions, the inhomogeneous zero field splittings, the effect of $P 1$ centers, and the hyperfine coupling from the nuclear spin, described as follows:

$$
H=H_{\text {flux }}+H_{\text {drive }}+H_{\text {ens }}+H_{\text {int }}
$$

The flux qubit Hamiltonian, interaction Hamiltonian, the $\mathrm{NV}^{-}$diamond ensemble Hamiltonian and the driving Hamiltonian can be written as:

$$
\begin{aligned}
H_{\text {flux }}= & \frac{\hbar}{2} \varepsilon \hat{\sigma}_{z}+\frac{\hbar}{2} \Delta \hat{\sigma}_{x} \\
H_{\text {drive }}= & \hbar \lambda \cos \omega t \cdot \hat{\sigma}_{z} \\
H_{\text {ens }}= & \hbar \sum_{k=1}^{N}\left\{D_{k} \hat{S}_{z, k}^{2}+E_{1}^{(k)}\left(\hat{S}_{x, k}^{2}-\hat{S}_{y, k}^{2}\right)+\right. \\
& \left.E_{2}^{(k)}\left(\hat{S}_{x, k} \hat{S}_{y, k}+\hat{S}_{y, k} \hat{S}_{x, k}\right)+g_{e} \mu_{B} \mathbf{B}_{\mathrm{NV}}^{(k)} \cdot \mathbf{S}_{\mathbf{k}}\right\} \\
H_{\text {int }}= & \hbar g_{e} \mu_{B} \hat{\sigma}_{z}\left(\sum_{k=1}^{N} \mathbf{B}_{\mathrm{qb}}^{(k)} \cdot \mathbf{S}_{k}\right)
\end{aligned}
$$

where $\widehat{\sigma}_{x, z}$ denotes the Pauli matrices for the flux qubit, $\widehat{\sigma}_{z}$ represents the population difference between two persistent current states in the flux qubit, $\Delta$ denotes the flux qubits tunneling energy, and $\varepsilon$ denotes the bias. From the Hamiltonian of the flux qubit, the frequency of the gap tunable flux qubit is calculated as $\omega_{F Q}=\sqrt{\varepsilon^{2}+\triangle^{2}}$. Since the bias $\varepsilon$ can be controlled by the external magnetic flux, we use the case of no bias (where the bias $\varepsilon$ is equal to 0 ), in which case the flux qubit has the best coherence time at the optimal point [23, 24. In the expression for the driving field of the flux qubit $H_{\text {drive }}, \lambda$ is the amplitude of the field, and $\omega$ is the angular frequency of the microwave. $H_{\text {ens }}$ represents the electron spin ensemble of $N$ individual $\mathrm{NV}^{-}$centers

with $\widehat{S}_{x, y, z}$ describing the electron spin 1 operators of the individual $\mathrm{NV}^{-}$center. $H_{\text {ens }}$ is characterized by the zero-field spitting $D$, the strain induced spitting $E$ and the Zeeman 
splitting term $g_{e} \mu_{B} B_{N V} \cdot S$. Here, $g_{e}=2$ is the $\mathrm{NV}^{-}$Lande factor and $\mu_{B}$ is Bohr's magneton. $B_{N V}$ is the magnetic field composed by the inhomogeneous magnetic field from $P 1$ centers and hyperfine induced magnetic field generated by nitrogen nuclear spins. $E_{1}^{(k)}$ denotes the strain along the x direction while $E_{2}^{(k)}$ denote the strain along the y direction. The term $B_{q b}$ in the $H_{\text {int }}$ presents the magnetic field generated by a flux-qubit persistent current.

Since it is difficult to solve the Hamiltonian described in the Eq. 1, our previous model considered the flux qubit and $\mathrm{NV}^{-}$centers as harmonic oscillators on the condition that the driving field strength of the flux qubit is weak [25]. We call this a many harmonic oscillator model (MHOM). The details of this model are discussed in the Appendix 5.3. Moreover, to obtain an intuitive explanation for the spectroscopic result, the Hamiltonian is further simplified with homogeneous $\mathrm{NV}^{-}$centers under a zero magnetic field (such as $D_{k}=D, B_{k}=B$ and $E_{k}=E$ ) without the driving term $(\lambda=0)$ [25]. Considering a homogeneous system, one can describe the NV ensemble by using just two harmonic oscillators. The Hamiltonian is rewritten as:

$$
H=\hbar \omega_{F Q} \hat{c}^{\dagger} \hat{c}+\hbar \omega_{b} \hat{b}^{\dagger} \hat{b}+\hbar \omega_{d} \hat{d}^{\dagger} \hat{d}+\hbar g\left(\hat{c}^{\dagger} \hat{b}+\hat{c} \hat{b}^{\dagger}\right)+\hbar J e^{i \theta} \hat{b}^{\dagger} \hat{d}+\hbar J e^{-i \theta} \hat{b} \hat{d}^{\dagger}
$$

where $\omega_{b}\left(\omega_{d}\right)$ denotes the frequency of the bright (dark) mode of the $\mathrm{NV}^{-}$centers, $\hat{c}^{\dagger}$

denotes a creation operator of the flux qubit, $\hat{b}^{\dagger}\left(\hat{d}^{\dagger}\right)$ denotes a creational operator of the bright (dark) mode of the $\mathrm{NV}^{-}$centers respectively, $g$ denotes the coupling strength between the $\mathrm{NV}^{-}$centers and the flux qubit, $J e^{i \theta}=g_{e} \mu_{B} B+i E$ denotes the coupling between the bright mode and dark mode. It is worth mentioning that the bright mode can be coupled with the flux qubit while the dark mode has no direct coupling with the flux qubit. The frequency of the bright state is assumed to be equal to that of the dark state, and so we have $\omega_{N V}=\omega_{b}=\omega_{d}=D$. This corresponds to the zero-field splitting of the $\mathrm{NV}^{-}$center between $|0\rangle$ and $| \pm 1\rangle$ states at zero magnetic field. (In the Appendix 5.4, the eigenvalues and eigenvectors of this simplified Hamiltonian are described, and we discuss the properties of them.)

\section{Main results}

\subsection{Master equation model (ME)}

A spectroscopy of this system was performed, and it was shown that power broadening due to the strong driving power is relevant in this system (shown in the [25]). There are several models attempting to understand this system. However, no existing model can explain the power broadening induced by strong microwave pulses for this system [25, 34]. It is important to build a model to understand this phenomenon. Such a model would contribute to evaluate the performance of the hybrid system under different driving powers and would help to optimize the fabrication parameters.

Here, we introduce our approach using a master equation model (ME) to study this hybrid system (shown in Fig.1). As the ensemble is driven by the flux qubit that is an effective single photon source, the number of excitations in the ensemble is much 
smaller than the number of electronic spins, and therefore it is reasonable to regard the spin ensemble as a number of harmonic oscillators as the previous authors considered. However, when the external driving power for the flux qubit is strong and induces the effect of the power broadening, we should consider the flux qubit as a two-level system and use the spin $1 / 2$ operator $\widehat{\sigma}$ instead of the harmonic oscillator operator. This is because strong driving power would populate higher energy structure in a harmonic oscillator system, which is different from the two-level system.

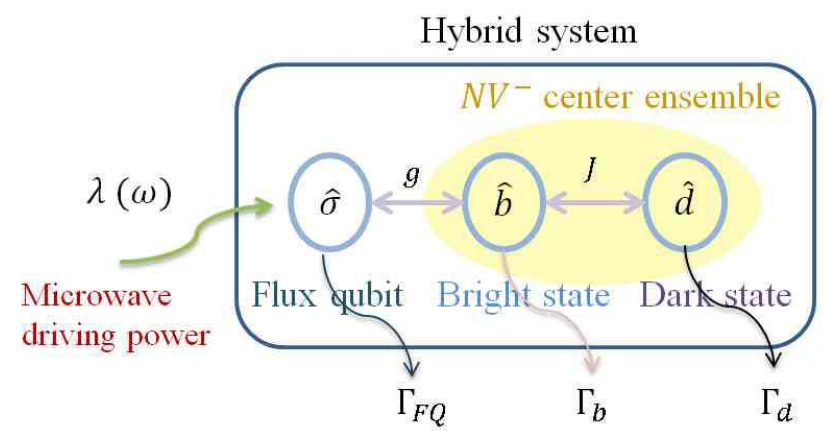

Figure 1. Schematic of the model for our approach (ME) to describe superconductor diamond hybrid system. The flux qubit is considered as a two level system. On the other hand, the NV centers are replaced by two harmonic oscillators, which we call a bright state mode and a dark state mode. The bright state is coupled with both the flux qubit and the dark state.

In such a case, the Hamiltonian should be given without harmonic oscillator approximation:

$$
\begin{aligned}
H & =\frac{1}{2} \hbar\left(\omega_{F Q}-\omega\right) \widehat{\sigma}_{z}+\hbar\left(\omega_{N V}-\omega\right) \hat{b}^{\dagger} \hat{b}+\hbar\left(\omega_{N V}-\omega\right) \hat{d}^{\dagger} \hat{d}+\hbar g\left(\widehat{\sigma}_{+} \hat{b}+\widehat{\sigma}_{-} \hat{b}^{\dagger}\right) \\
& +\hbar J e^{i \theta} \hat{b}^{\dagger} \hat{d}+\hbar J e^{-i \theta} \hat{b} \hat{d}^{\dagger}+\frac{\lambda}{2} \widehat{\sigma}_{x}
\end{aligned}
$$

Moreover, we use the dissipative term of Lindblad equation [37, 38, 39] to present the effect of inhomogeneous broadening induced by the strain and Zeeman splitting from each $\mathrm{NV}^{-}$center:

$$
\begin{aligned}
\frac{\partial \rho}{\partial t}= & -\frac{i}{\hbar}[\rho, H]+L(\rho) \\
L(\rho) & =-\Gamma_{F Q}\left(\widehat{\sigma}_{+} \widehat{\sigma}_{-} \rho+\rho \widehat{\sigma}_{+} \widehat{\sigma}_{-}-2 \widehat{\sigma}_{-} \rho \widehat{\sigma}_{+}\right) \\
& -\Gamma_{b}\left(\hat{b}^{\dagger} \hat{b} \rho+\rho \hat{b}^{\dagger} \hat{b}-2 \hat{b} \rho \hat{b}^{\dagger}\right)-\Gamma_{d}\left(\hat{d}^{\dagger} \hat{d} \rho+\rho \hat{d}^{\dagger} \hat{d}-2 \hat{d} \rho \hat{d}^{\dagger}\right)
\end{aligned}
$$

where $L(\rho)$ denotes a Lindblad super-operator and $\rho$ is the density matrix. $\Gamma_{F Q}, \Gamma_{b}$, and $\Gamma_{d}$ denote the decay rate of the flux qubit, bright state, and dark state, respectively. The inhomogeneous broadening induces unknown phases on the bright state (or dark state), which transforms the collective mode into another orthogonal modes [24, 25, 34]. It has been shown that an inhomogeneous distribution of the frequency can be considered as an energy relaxation of the collective mode [35], which can be described as a Markovian 
process. Actually, an exponential decay of the collective mode in this hybrid system has been observed in [24], which is consistent with the model of the Markovian noise. So, to include the effect of inhomogeneous broadening, we add the Markovian relaxation terms of the bright state and the dark state in the Lindblad equation.

\subsection{Three Harmonic Oscillator model (THOM)}

In order to describe our sample of the flux qubit coupled with the $\mathrm{NV}^{-}$centers, we have already obtained the necessary parameters $(\zeta, \delta B, \delta E, \delta D)$ for the simulation of the MHOM from the experiment [25]. However, as we describe before, this model will be invalid for a strong driving regime, and we should use the ME when the power broadening is relevant. Since we use a different model, we cannot straightforwardly use the parameters that are used in MHOM. We need to find suitable parameter

set $\left(g, J, \Gamma_{F Q}, \Gamma_{b}, \Gamma_{d}\right)$ for the ME. For this purpose, it is convenient to have a fitting function that let us find out such a new parameter set $\left(g, J, \Gamma_{F Q}, \Gamma_{b}, \Gamma_{d}\right)$ from the known parameter set $(\zeta, \delta B, \delta E, \delta D)$. In the condition of weak excitation, the excited probability of the qubit is described as

$$
\left\langle\hat{\sigma}_{+} \hat{\sigma}_{-}\right\rangle=\left(\frac{\lambda}{2}\right)^{2} \times\left|\frac{\left[\left(i \Gamma_{b}-\omega_{b}^{\prime}\right)\left(i \Gamma_{d}-\omega_{d}^{\prime}\right)-J^{2}\right]}{\left(i \Gamma_{c}-\omega_{c}^{\prime}\right)\left[\left(i \Gamma_{b}-\omega_{b}^{\prime}\right)\left(i \Gamma_{d}-\omega_{d}^{\prime}\right)-J^{2}\right]-g^{2}\left(i \Gamma_{d}-\omega_{d}^{\prime}\right)}\right|^{2}
$$

where $\omega_{F Q}^{\prime}=\omega_{F Q}-\omega$ and $\omega_{N V}^{\prime}=\omega_{N V}-\omega$. We can derive this from a three harmonic oscillator model (THOM) where the flux qubit is regarded as a harmonic oscillator (See the Appendix 5.5 for the details). It is worth mentioning that this analytical solution is useful only for weak driving limit. However, this approach plays a crucial role to find the parameter set for the master equation approach, as we describe later.

\subsection{Determination of parameters for $M E$}

In this section, we will introduce the process about how to determine the necessary parameters to describe our devices for our ME. We have already obtained the parameters to describe our devices for the MHOM (the model described in the paper [25]):

$$
\delta B / 2 \pi=0.056 \mathrm{mT} ; \delta \mathrm{E} / 2 \pi=4.4 \mathrm{MHz} ; \delta \mathrm{D} / 2 \pi=0.2 \mathrm{MHz} ;
$$

We construct a scheme to translate the parameters $(\zeta, \delta B, \delta E, \delta D)$ in the MHOM into the parameters in our new approach $\left(g, J, \Gamma_{b}, \Gamma_{d}\right)$, which is one of the main results in our paper. As mentioned before, THOM provides the fitting function to obtain such parameters. If we fit the MHOM spectrum by this function, it seems that we might determine the parameters needed in the ME because THOM is equivalent to ME for a weak driving regime. However, due to many parameters to be estimated, if we naively apply the fitting function to the MHOM, we cannot obtain a unique set of parameters from the fitting result. In general, it requires other methods to fix the set of parameters, and we have developed such method. Firstly, from the $T 1$ measurement, we estimate the value of the flux qubit decay rate $\Gamma_{F Q}$. It is worth mentioning that $\Gamma_{F Q}$ denotes a decay rate of the flux qubit for both MHOM and ME so that we could use the same 
value for $\Gamma_{F Q}$ in the $\mathrm{ME}$ as that used in $\operatorname{MHOM}\left(\Gamma_{F Q}=\frac{1}{2 T_{1}}=0.33 \mathrm{MHz}\right)$. Secondly, by using the expression of eigenvalues and eigenvectors of the Hamiltonian described in Eq. 7, we find the method to estimate the values of $J$ and $g$ from MHOM. Finally, by fixing the values of $J, g$, and $\Gamma_{F Q}$, we use the analytical solution of THOM to fit the MHOM's result and determine the other two parameters of $\Gamma_{b}$ and $\Gamma_{d}$. The detail of the procedure is described as follows.

3.3.1. Estimation of the coupling strength $J$ and $g$ We explain how to determine the coupling strength $J$ and $g$ from the MHOM.

First, by the Hamiltonian in the Eq. 7, the frequency difference between the right peak and left peak on the resonant condition depends on the coupling strength $J$ and $g$ $(\delta=0)$, as shown in the Appendix 5.4. The relationship is described as

$$
\left(E_{\text {right }}-E_{\text {left }}\right) / \hbar=2 \sqrt{g^{2}+J^{2}}
$$

Also, if we assume $g \gg \Gamma_{b}, \Gamma_{d}, \Gamma_{c}$ in the Eq. 10, we can calculate the frequency difference between the left and right peak

$$
\left(E_{\text {right }}-E_{\text {left }}\right) / \hbar \simeq \sqrt{4\left(g^{2}+J^{2}\right)-\left(\Gamma_{b}-\Gamma_{d}\right)^{2}} \simeq 2 \sqrt{g^{2}+J^{2}}
$$

and this is consistent with the Eq. 12. Moreover, from the frequency difference between the left and right peak plotted by MHOM (shown in Fig. 8), we obtain the following relationship

$$
\left(E_{\text {right }}-E_{\text {left }}\right) / \hbar \simeq 27 \mathrm{MHz} \Longrightarrow \sqrt{\mathrm{g}^{2}+\mathrm{J}^{2}} \simeq 13.5 \times 2 \pi \mathrm{MHz}
$$

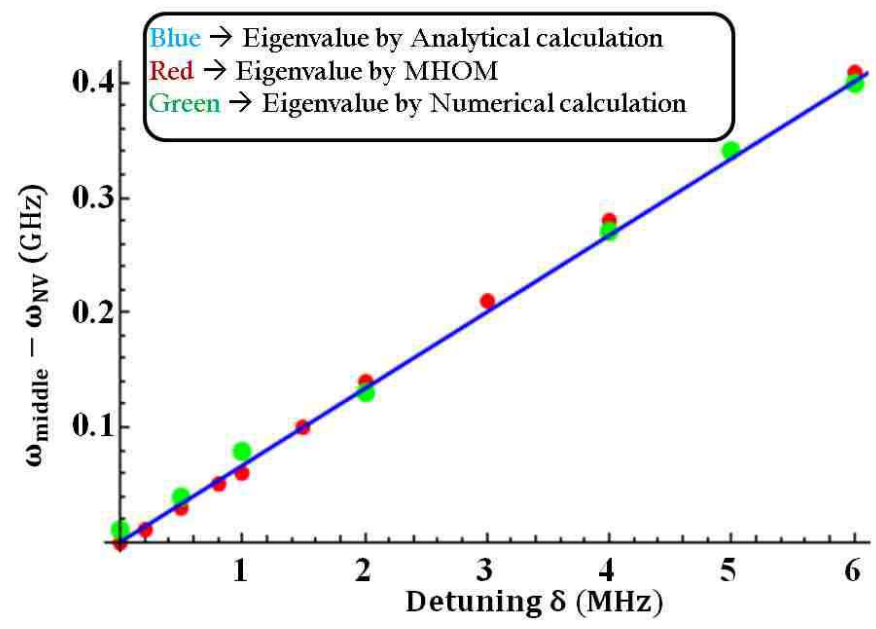

Figure 2. Energy shift of the middle peak in the spectroscopy against the detuning. Here, the red dots represent the simulation result with the MHOM, and the green dots represent numerically calculated eigenvalues. The blue line represents an analytical solution using a perturbation theory.

Second, we use a relationship between the middle peak frequency and detuning $\delta$. We have shown that the middle peak frequency of the spectroscopy will be shifted by adding the energy detuning, and the energy shift increases linearly as we enlarge 
the detuning when the detuning is considered as a perturbation $\left(\delta \ll \omega_{N V}\right)$ (See the Appendix 5.5). This is described as

$$
\omega_{\text {middle }}-\omega_{N V} \simeq \frac{\delta J^{2}}{g^{2}+J^{2}}
$$

where the proof is described in the Appendix 5.5. Actually, we plot the energy shift of the middle peak against the detuning by solving MHOM (shown in Fig.21), and we fit this plot by Eq. 15. From this fitting, we obtain the following relationship between the parameters for ME:

$$
\frac{J^{2}}{g^{2}+J^{2}} \approx 0.067 \Longrightarrow J \simeq 0.27 g
$$

However, since we use a perturbation to obtain the fitting function in Eq,15, this result would be invalid for a large detuning. To confirm the validity of the perturbation, we numerically calculate the eigenvalues of the Hamiltonian in the Eq. 7, and plot the results in Fig. 2. Since we have a good agreement between perturbation calculation and numerical results, we conclude that the perturbation is valid in this parameter regime.

By combining the Eq. 14 and Eq. 16, we can estimate the values of J and $g$ :

$$
J \simeq 3.5 \times 2 \pi \mathrm{MHz} ; \mathrm{g} \simeq 13 \times 2 \pi \mathrm{MHz} ;
$$

3.3.2. Estimation of decay rate $\Gamma_{b}$ and $\Gamma_{d}$ The value of $\Gamma_{b}$ mainly affects the width of the side peaks while the width of the middle peak is determined by the value of $\Gamma_{d}$ (See the Appendix 5.5 for the details). This means that, we can use the THOM as a fitting function to fit the spectrum reproduced by the MHOM if the parameters $\Gamma_{F Q}, J$ and $g$ are known, and we have obtained the following parameters

$$
\Gamma_{d} \simeq 0.49 \times 2 \pi \mathrm{MHz} ; \Gamma_{\mathrm{b}} \simeq 6.4 \times 2 \pi \mathrm{MHz} ;
$$

Therefore, all necessary parameters for the ME have been estimated by our scheme.

\subsection{Reproducing experimental results with the $M E$}

With these parameters, we use the ME to reproduce the spectroscopic measurement in experiment for different driving power (See Fig,3 and Fig,4). Not only in the weak power case but also in the strong driving power case, there is a good agreement between simulation and experiment, as shown in Fig.3 and Fig.4. Thus, our new approach with the ME is shown to be useful to reproduce the spectroscopy even when the power broadening is relevant.

Furthermore, in order to compare the theory and experiment in detail, we analyze the width of the middle peak which can be well fitted by a Lorentz function.

$$
\frac{a \gamma^{2}}{\left(\omega-\omega^{\prime}\right)^{2}+\gamma^{2}}+c
$$

where $\gamma$ correspond to the width of the Lorentz curve and $\omega^{\prime}$ is the center frequency, $a$ and $c$ and the fitting coefficients. We use the Lorentzian function to fit the middle peak 

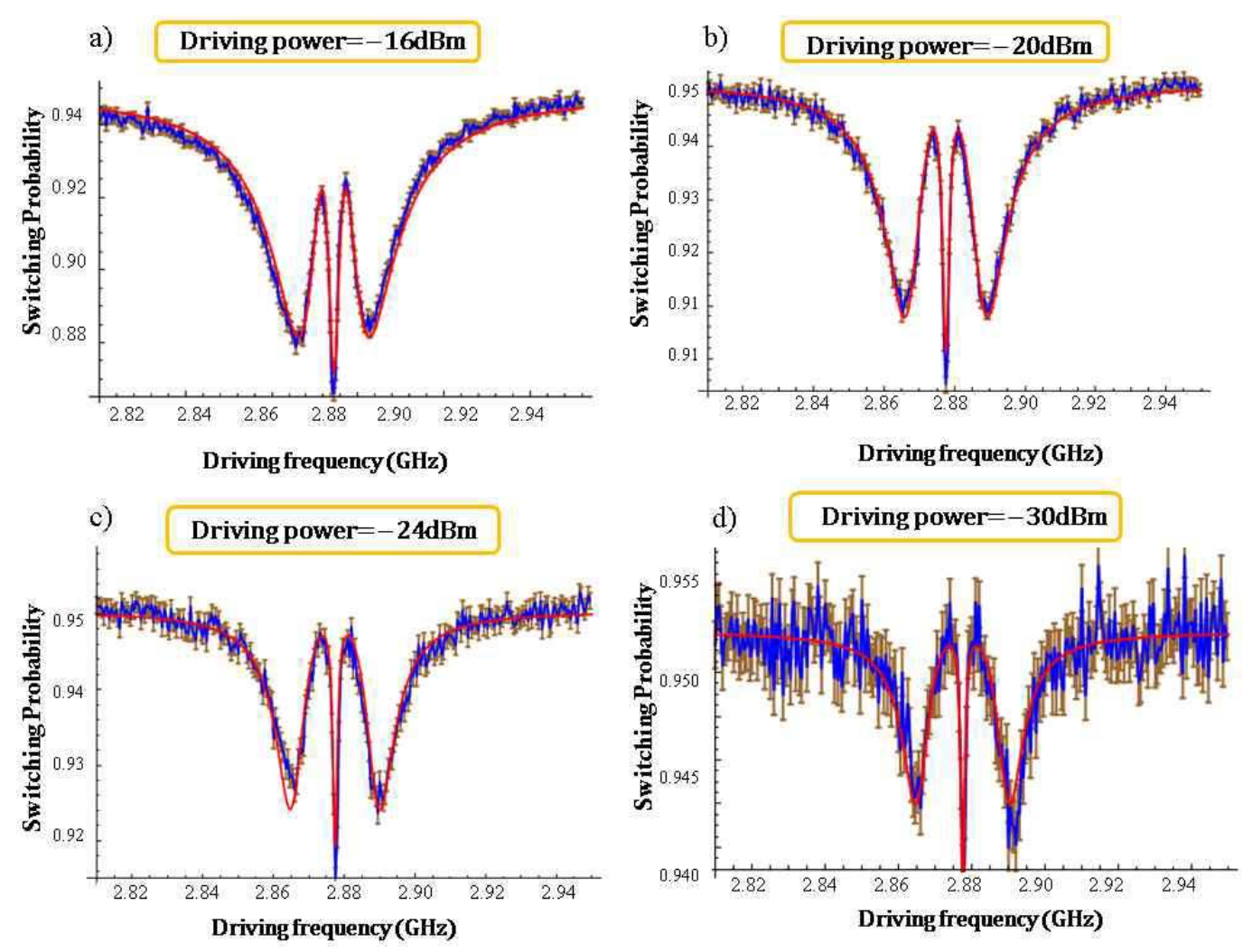

Figure 3. Spectroscopy with different driving power. The blue dots are the measured data where continuous lines are drawn through the points as a guide to the eye. The brown line denotes the error bar. The red curve shows the numerical results by solving the ME. For the simulation, we use parameters of $\omega_{F Q}=\omega_{N V}=2.878 \times 2 \pi \mathrm{GHz}$, $g=12.95 \times 2 \pi \mathrm{MHz}, J=3.46 \times 2 \pi \mathrm{MHz}, \Gamma_{F Q}=0.300 \times 2 \pi \mathrm{MHz}, \Gamma_{d}=0.493 \times 2 \pi$ $\mathrm{MHz}$, and $\Gamma_{b}=6.433 \times 2 \pi \mathrm{MHz}$. Here, $a, b, c$, and $d$ present the spectrum in different driving power: $-16 \mathrm{dBm},-20 \mathrm{dBm},-24 \mathrm{dBm}$ and $-30 \mathrm{dBm}$, respectively. It is worth mentioning that we cannot observe a power broadening around $-30 \mathrm{dBm}$ as described in Fig. 5, and so the power is sufficiently weak in this regime.

calculated by the ME (shown in Fig 5 a). By the fitting, we obtain the FWHM (Full Width at Half Maximum) $2 \gamma$ with the ME, MHOM, and the experiments, respectively. In the Fig馬(b), we plot them, and the power broadening is clearly observed in both the experiment and the ME, while MHOM cannot describe the power broadening. These results also show that the ME surpasses the MHOM in order to reproduce the experiments.

\section{Conclusion}

In summary, we introduce a new theoretical approach to describe spectroscopic measurements of a superconducting flux qubit coupled with $\mathrm{NV}^{-}$centers in diamond. Although previous models are applicable only when the driving power of the applied microwave is weak, we have succeeded to reproduce the experimental spectroscopy even when the power broadening becomes relevant due to the strong driving. Since it is 
a)

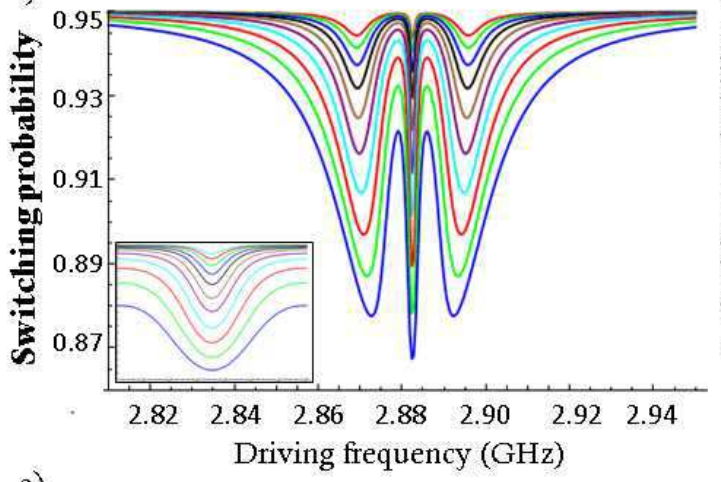

c)

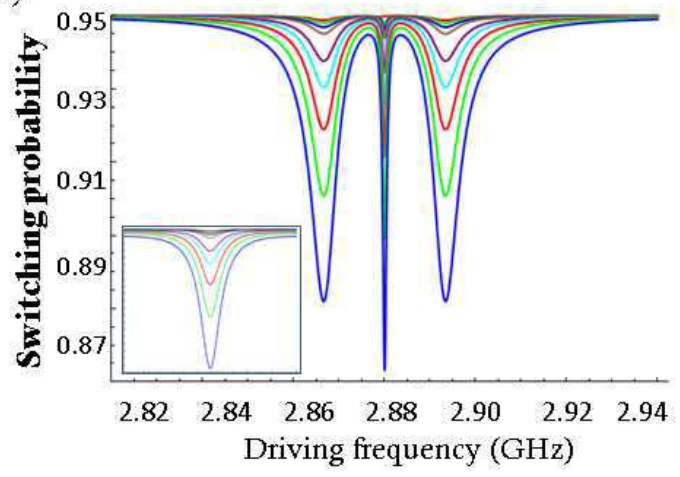

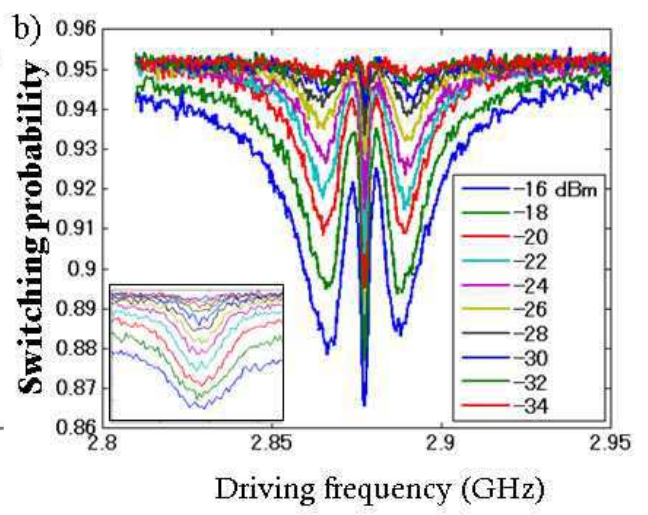

Filename: fig16a

Figure 4. The spectrum with power broadening. (a) Power dependence of the energy spectrum of our hybrid system obtained from the experiment. (b) Numerical results of the energy spectrum by solving ME. (c) Numerical results of the energy spectrum by solving MHOM. We use the same parameters as those in Fig 3 Inset of each picture is the enlargement of the spectrum over the middle peak region.

a)

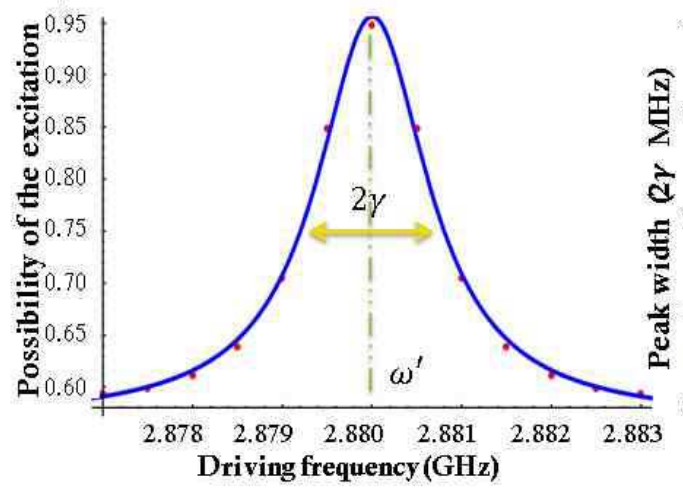

b)

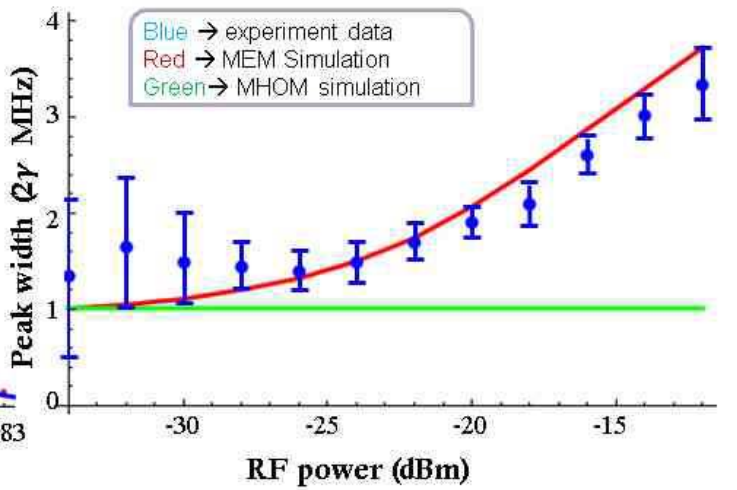

Figure 5. Behavior of the width of the middle peak with different driving power. (a) The middle peak is fit by the Lorentz function. The red dots denote the simulation result from ME. The blue curve denotes the fitting result of a Lorentz function. The parameters for ME are the same as Fig 16. (b) The full width at half maximum (FWHM) with different driving power. The blue dots present the FWHM from the experiment with error bar. The red line denotes the FWHM from the ME by Lorentz fitting. The green line is the FWHM resulted from MHOM simulation. 
typically difficult to remove the effect of the power broadening in the spectroscopy when a superconducting flux qubit is driven by the microwave, our results provide an efficient way to analyze the superconductor diamond hybrid system. Our method will be useful to characterize this system for the application of quantum information processing.

\section{Acknowledgments}

This work was supported by KAKENHI(S) 25220601. The research results have been achieved by the Commissioned Research of National Institute of Information and Communications Technology (NICT), JAPAN.

\section{Appendix}

\subsection{Experimental setup}

We briefly describe the experimental setup. The hybrid system is shown in Fig 6, The $\mathrm{NV}^{-}$ensemble is generated by an ion implantation and annealing in vacuum [34]. The density of the $\mathrm{NV}^{-}$centers is approximately $5 \times 10^{17} \mathrm{~cm}^{-3}$. The diamond including the $\mathrm{NV}^{-}$centers is glued on the top of the flux qubit where the distance between the flux qubit and the surface of the $\mathrm{NV}^{-}$diamond is less than $1 \mu \mathrm{m}$. We can control the hybrid system by the microwave, and this system is measured by switching current in the SQUID that is inductively coupled with the flux qubit.

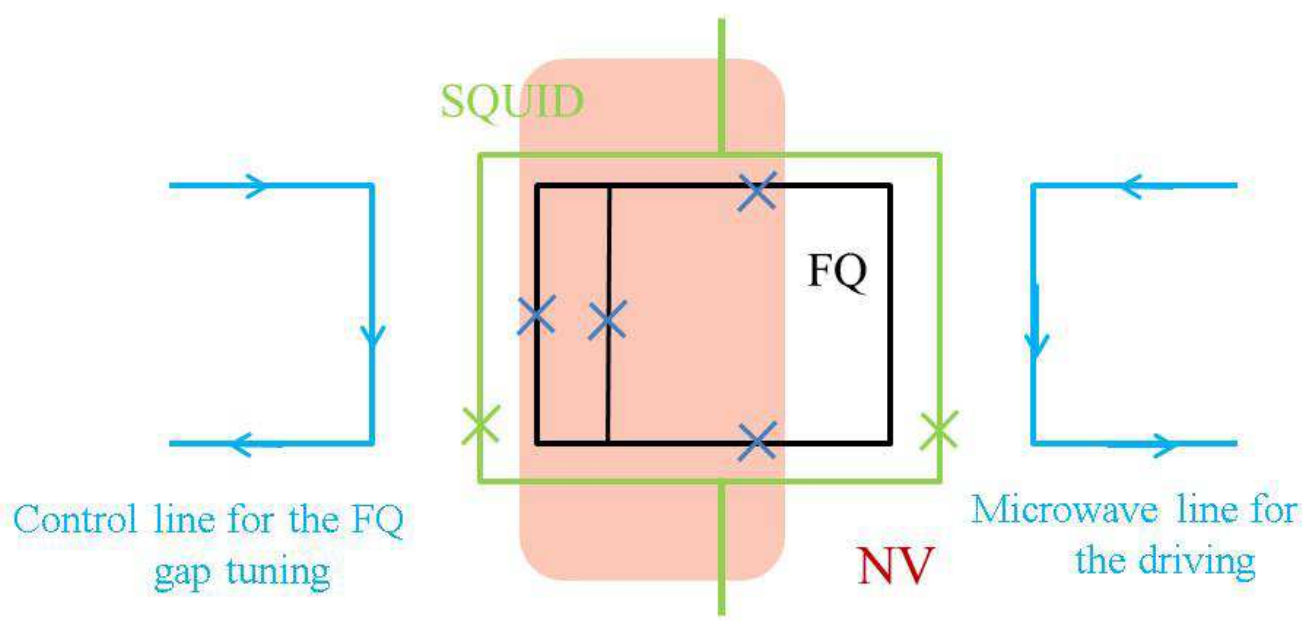

Figure 6. Illustration of the hybrid system. It contains the SQUID, the control line, microwave line, a flux qubit with four junctions (the cross in the diagram), and an ensemble of NV center in the diamond that is glued on the top of the flux qubit 34. 


\subsection{Many Harmonic Oscillator model (MHOM)}

There are two groups that performed spectroscopy of a superconductor-diamond hybrid system under zero external magnetic field [21, 25, 34, and both groups observed two broader side peaks and a middle sharp peak in the spectrum. However, in the papers [21, 34, such three peaks could not be reproduced by the theoretical model. In a paper [25], such three peaks are firstly reproduced by a numerical simulation based on a new model.

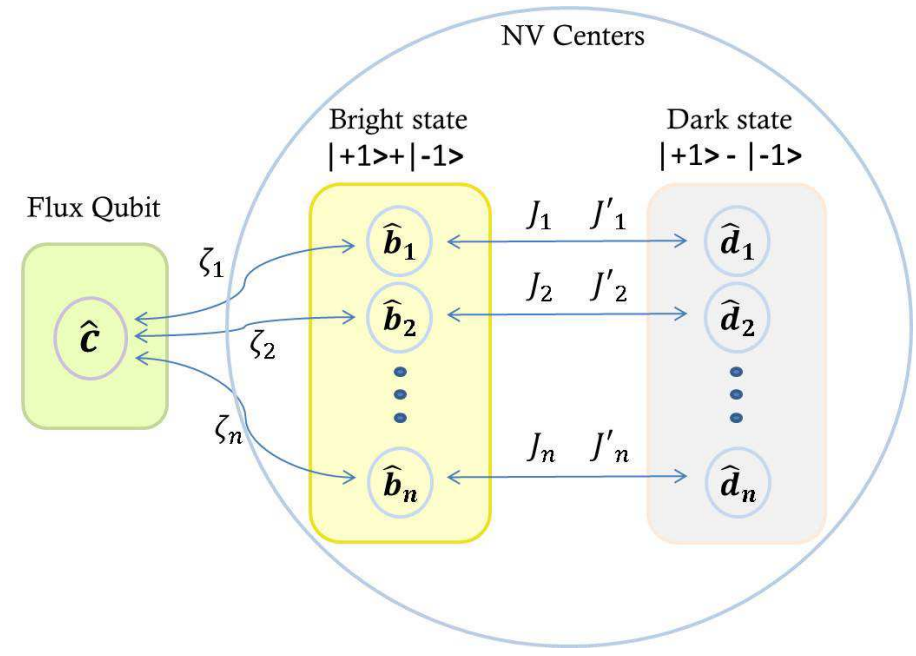

Figure 7. The schematic of the MHOM. Here, $\hat{c}$ denotes the flux qubit, $\omega^{(k)}{ }_{b}\left(\omega^{(k)}{ }_{d}\right)$ denotes the frequency of the $k$ th bright state (a dark state) in $\mathrm{NV}^{-}$centers, $\hat{b}_{k}^{\dagger}\left(\hat{d}_{k}^{\dagger}\right)$ denotes the bright state (dark state) of the $k$ th $\mathrm{NV}^{-}$centers, $J_{k}$ denotes the effect from Zeeman splitting, $J_{k}^{\prime}$ denotes the effect from the strain, and $\zeta_{k}$ denotes the coupling between the flux qubit and $k$ th $\mathrm{NV}^{-}$centers.

We explain how to obtain the model introduced in [25]. In order to solve the full Hamiltonian described in Eq. 1, the flux qubit and $\mathrm{NV}^{-}$centers are regarded as harmonic oscillators on the condition that the driving field strength of the flux qubit is weak [25]. In this case, the average number of the excitation at the flux qubit and $\mathrm{NV}^{-}$ center is much less than one. Since there are two types of excited states in the $\mathrm{NV}^{-}$ center ensemble, two creation operators (such as $\widehat{b}^{+}$and $\widehat{d}^{+}$) are defined to describe the properties of $\mathrm{NV}^{-}$centers (shown in Fig.7). Here, $\widehat{b}^{+}$denotes a creation operator of the bright states that can be directly coupled with the flux qubit, while $\widehat{d}^{+}$denotes a creation operator of the dark states that interact only indirectly with the flux qubit via the bright states. Then making the rotating wave approximation, we define the many harmonic oscillator model (MHOM) and obtain the Hamiltonian as following:

$$
\begin{aligned}
& H=\hbar\left(\omega_{F Q}-\omega\right) \hat{c}^{\dagger} \hat{c}+\hbar \frac{\lambda}{2}\left(\hat{c}+\hat{c}^{\dagger}\right) \\
& +\sum_{k=1}^{N}\left[\hbar\left(\omega_{b}^{(k)}-\omega\right) \hat{b}_{k}^{\dagger} \hat{b}_{k}+\hbar\left(\omega_{d}^{(k)}-\omega\right) \hat{d}_{k}^{\dagger} \hat{d}_{k}+\hbar \zeta_{k}\left(\hat{c}^{\dagger} \hat{b}_{k}+\hat{c} \hat{b}_{k}^{\dagger}\right)\right. \\
& \left.+\hbar\left(J_{k}+i J_{k}^{\prime}\right) \hat{b}_{k}^{\dagger} \hat{d}_{k}+\hbar\left(J_{k}-i J_{k}^{\prime}\right) \hat{b}_{k} \hat{d}_{k}^{\dagger}\right]
\end{aligned}
$$


where $\omega^{(k)}{ }_{b}=D_{k}-E_{1}^{(k)}$ denotes a frequency of the $k$ th bright state and $\omega^{(k)}{ }_{d}=D_{k}+E_{1}^{(k)}$ denotes a frequency of the $k$ th dark state. Since the effect of the the Zeeman splitting (strain) allows the transition between the bright states and dark states, a coupling strength of $J_{k}=g_{e} \mu_{B} B_{k}\left(J_{k}^{\prime}=E_{2}^{(k)}\right)$ is defined to present the interaction between them.

Similarly, the two-level system of the flux qubit is simplified as a harmonic oscillator, and so this can be described by the creational operator $\widehat{c}^{+}$. The flux qubit is coupled only with one mode of the ensemble (the bright states) and the individual coupling strength is presented by the value of $\zeta$. $\lambda$ denotes the driving power of the flux qubit while $\omega$ denotes the frequency of the driving power.

\subsection{Heisenberg-Langevin equations of MHOM}

We explain the details about how we can solve the model introduced in [25], which we call MHOM. Based on the Hamiltonian in Eq. 20, the Heisenberg-Langevin equations of the system are written as [25]:

$$
\begin{aligned}
\frac{d}{d t} \widehat{c} & =-\left(\Gamma_{F Q}+i \omega_{F Q}\right) \hat{c}-i\left(\sum_{k=1}^{N} \zeta_{k} \cdot \hat{b}_{k}\right)-i \frac{\lambda}{2} \\
\frac{d}{d t} \widehat{b}_{k} & =-\left(\Gamma_{b}+i \omega_{b}^{(k)}\right) \hat{b}_{k}-\left(i J_{k}+J_{k}^{\prime}\right) \hat{d}_{k}-i \zeta_{k} \cdot \hat{c} \\
\frac{d}{d t} \widehat{d}_{k} & =-\left(\Gamma_{d}+i \omega_{d}^{(k)}\right) \hat{d}_{k}-\left(i J_{k}+J_{k}^{\prime}\right) \hat{b}_{k}
\end{aligned}
$$

where $\Gamma_{F Q}, \Gamma_{b}, \Gamma_{d}$ denote the energy decay of the flux qubit and the $\mathrm{NV}^{-}$center bright state and dark state, respectively. By transforming these into Fourier space, then we obtained the expression of $\hat{c}$ :

$$
\hat{c}=\frac{\lambda}{2} \times \frac{1}{\omega-\omega_{F Q}+i \Gamma_{F Q}-\left(\sum_{k=1}^{N}\left|\zeta_{k}\right|^{2} \frac{\omega-\omega_{d}^{(k)}+i \Gamma_{d}}{\left(\omega-\omega_{b}^{(k)}+i \Gamma_{b}\right)\left(\omega-\omega_{d}^{(k)}+i \Gamma_{d}\right)-\left(J_{k}^{2}+J^{\prime 2}\right)}\right)}
$$

These Heisenberg-Langevin equations was solved to obtain $\left\langle\hat{c}^{\dagger} \hat{c}\right\rangle$, which denotes the average number of the excitation in the flux qubit [25]. It is worth mentioning that the main decoherence source of this hybrid system is inhomogeneous broadening $[21,22,23,24,20,29,30,31,32$, and the Heisenberg-Langevin equations used in [25] include such effect. In this model, the spectroscopic measurements of the flux qubit with $\mathrm{NV}^{-}$centers are reproduced with a narrow peak located in the middle of the avoid crossing (shown in Fig. 8). Two broad resonances are observed on the side, which we call a left peak and a right peak, respectively. Also, one sharp resonance is observed between them around $2.88 \mathrm{GHz}$, which we call a middle peak. These agree with the experimental results in the limit of weak driving [21, 25, 34].

\subsection{Eigenvector and Eigenvalue}

To understand the spectrum shown in Fig. 8, the Hamiltonian in Eq. 66 is diagonalised in [25], and we explain the results here.

$$
H=\hbar \omega_{F Q} \hat{c}^{\dagger} \hat{c}+\hbar \omega_{N V} \hat{b}^{\dagger} \hat{b}+\hbar \omega_{N V} \hat{d}^{\dagger} \hat{d}+\hbar g\left(\hat{c}^{\dagger} \hat{b}+\hat{c} \hat{b}^{\dagger}\right)+\hbar J\left(e^{i \theta} \hat{b}^{\dagger} \hat{d}+e^{-i \theta} \hat{b} \hat{d}^{\dagger}\right)
$$




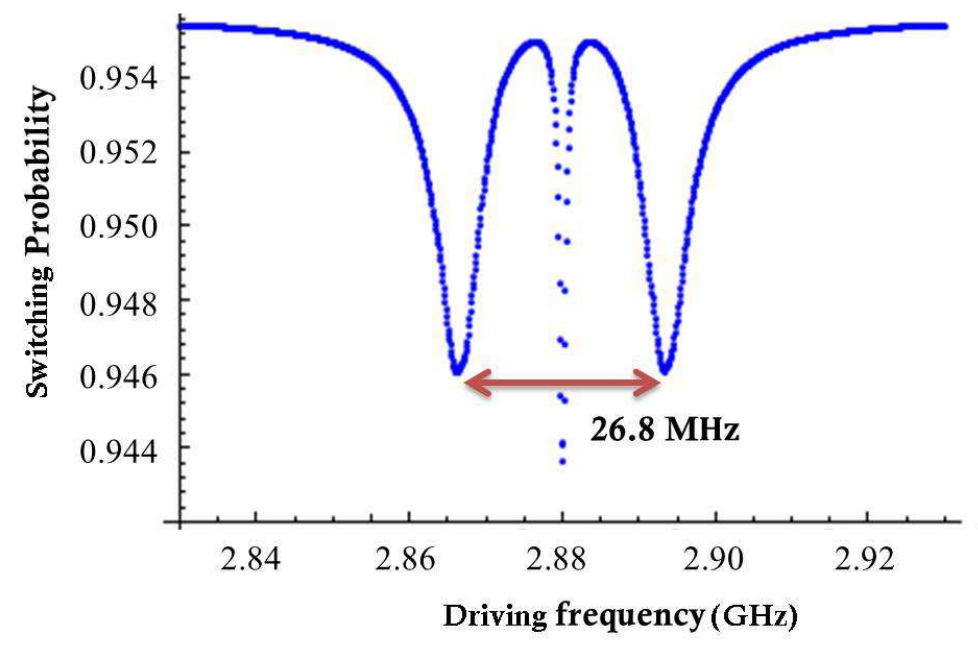

Figure 8. The energy spectrum of the hybrid system by MHOM in 25]. Here, $\mathrm{x}$ axis denotes the microwave driving frequency and $\mathrm{y}$ axis denotes the switching probability of the SQUID which corresponds to the microwave absorption of the flux qubit $\left(P_{\text {switching }}=1-P_{\text {absorption }}\right)$. The spectrum is plotted under the conditions of $g_{e} \mu_{B} B / 2 \pi=28 \mathrm{MHz} ; N=36000 ; \omega_{F Q} / 2 \pi=\omega_{b} / 2 \pi=\omega_{d} / 2 \pi=2.88 \mathrm{GHz}$; $\delta\left(g_{e} \mu_{B} B / 2 \pi\right)=3.1 \mathrm{MHz}(\mathrm{FWHM}) ; \delta(E / 2 \pi)=4.4 \mathrm{MHz}(\mathrm{FWHM}) ; \Gamma_{F Q} / 2 \pi=0.3$ $\mathrm{MHz}(\mathrm{FWHM}) ; \Gamma_{N V} / 2 \pi=\delta(D / 2 \pi)=0.2 \mathrm{MHz}(\mathrm{FWHM}) ; \lambda / 2 \pi=20 \mathrm{MHz}$.

where $g$ denotes the collective coupling strength between the bright state and the flux qubit, J denotes the coupling strength between the bright state and the dark state, and

\section{FQ coupled with NV}

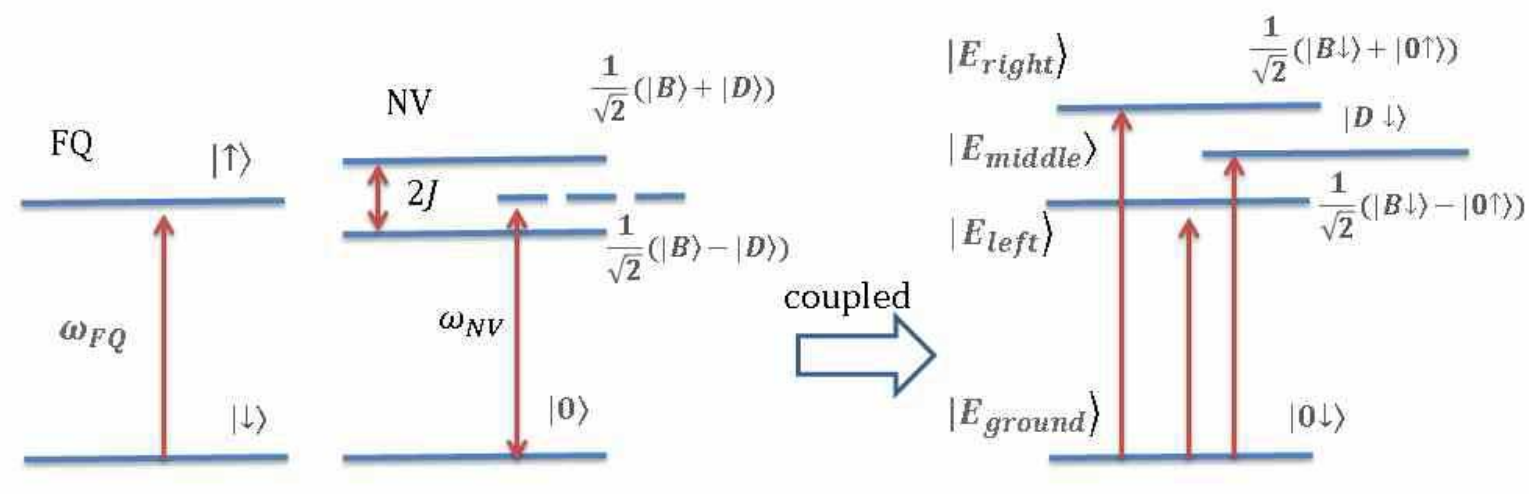

Figure 9. The energy diagram. In the picture, the left side denotes the energy diagram without coupling (coupling strength $g=0$ ) while the right side denotes the case with coupling. Here we assume that the coupling strength between the dark state and bright state is much smaller than the coupling strength between the flux qubit and $\mathrm{NV}^{-}$center $(J \ll g)$.

$|0\rangle$ denotes the ground state of $\mathrm{NV}^{-}$center. Since $\mathrm{NV}^{-}$center is a spin 1 system, it has 
three electron states: $|0\rangle,|1\rangle$ and $|-1\rangle$. Suppose that $|B\rangle$ and $|D\rangle$ denote the excited states of bright state and dark state respectively where

$$
\begin{aligned}
& |B\rangle=\frac{1}{\sqrt{2}}(|1\rangle+|-1\rangle) \\
& |D\rangle=\frac{1}{\sqrt{2}}(|1\rangle-|-1\rangle) .
\end{aligned}
$$

$|\downarrow\rangle$ and $|\uparrow\rangle$ denote the ground state and excited state of the flux qubit. The relationship between the ground state and the excited state is described as follows:

$$
\begin{aligned}
& \hat{b}^{\dagger}|0\rangle_{N V}=|B\rangle_{N V} \\
& \hat{d}^{\dagger}|0\rangle_{N V}=|D\rangle_{N V} \\
& \hat{c}^{\dagger}|\downarrow\rangle_{F Q}=|\uparrow\rangle_{F Q} ;
\end{aligned}
$$

The transition from the ground state to the first excited state (shown in Fig 9), which corresponds to the left side peak in the experimental spectroscopy (shown in Fig 8 ), is described by:

$$
\begin{aligned}
& \triangle E_{\text {left }}=E_{\text {left }}-E_{0}=\hbar\left(\omega_{N V}-\sqrt{g^{2}+J^{2}}\right) \\
& \left|E_{\text {left }}\right\rangle=\left(-\frac{1}{\sqrt{2}}|B \downarrow\rangle+\frac{1}{\sqrt{2}} \frac{g}{\sqrt{g^{2}+J^{2}}}|0 \uparrow\rangle\right)+\frac{1}{\sqrt{2}} \frac{J e^{-i \theta}}{\sqrt{g^{2}+J^{2}}}|D \downarrow\rangle
\end{aligned}
$$

The transition from the ground state to the second excited state (shown in Fig 9), which corresponds to the middle peak of the spectroscopy (shown in Fig.8), is described by:

$$
\begin{aligned}
& \triangle E_{\text {middle }}=E_{\text {middle }}-E_{0}=\hbar \omega_{N V} \\
& \left|E_{\text {middle }}\right\rangle=-\frac{J e^{i \theta}}{\sqrt{g^{2}+J^{2}}}|0 \uparrow\rangle+\frac{g}{\sqrt{g^{2}+J^{2}}}|D \downarrow\rangle
\end{aligned}
$$

The transition from the ground state to the third excited state (shown in Fig 9), which corresponds to the right side peak of the experimental spectroscopy (shown in Fig,8), is described by:

$$
\begin{aligned}
& \triangle E_{\text {right }}=E_{\text {right }}-E_{0}=\hbar\left(\omega_{N V}+\sqrt{g^{2}+J^{2}}\right) \\
& \left|E_{\text {right }}\right\rangle=\left(\frac{1}{\sqrt{2}}|B \downarrow\rangle+\frac{1}{\sqrt{2}} \frac{g}{\sqrt{g^{2}+J^{2}}}|0 \uparrow\rangle\right)+\frac{1}{\sqrt{2}} \frac{J e^{-i \theta}}{\sqrt{g^{2}+J^{2}}}|D \downarrow\rangle
\end{aligned}
$$

Due to the hybridization induced by the coupling between the flux qubit and $\mathrm{NV}^{-}$ centers, the energy level of the exited states of the hybrid system is split into three levels. The left and right peaks in the Fig.(8) correspond to the first and third excited states which contain a bright state of the $\mathrm{NV}^{-}$centers. We call these two exited states as the hybrid bright states. The middle peak correspond to the second excited state that shows the existence of a $\mathrm{NV}^{-}$dark state, which we call the hybrid dark state. Interestingly, the second excited state contains $|0 \uparrow\rangle$ where the flux qubit is excited so that the signal of this excited state can be detected via the spectroscopic measurement of the flux qubit. 


\subsection{Properties of THOM}

We explain how to solve THOM, and discuss the properties of this model.

5.5.1. Solution of THOM In the condition of weak excitation, we can use a harmonic oscillator to replace the spin $1 / 2$ operator $\left(\widehat{\sigma}_{+} \Rightarrow \hat{c}^{\dagger}\right)$ and use two harmonic oscillators $\hat{b}^{\dagger}$ and $\hat{d}^{\dagger}$ to represent $\mathrm{NV}^{-}$center. Then, we use the non-hermitian decay terms $\left(i \Gamma_{c} \hat{c}^{\dagger} \hat{c}\right.$, $\left.i \Gamma_{b} \hat{b}^{\dagger} \hat{b}, i \Gamma_{d} \hat{d}^{\dagger} \hat{d}\right)$ to take the role of Lindblad terms, presenting the relaxation in the flux qubit, bright state and dark state (shown in Fig.10). We rewrite the Hamiltonian as

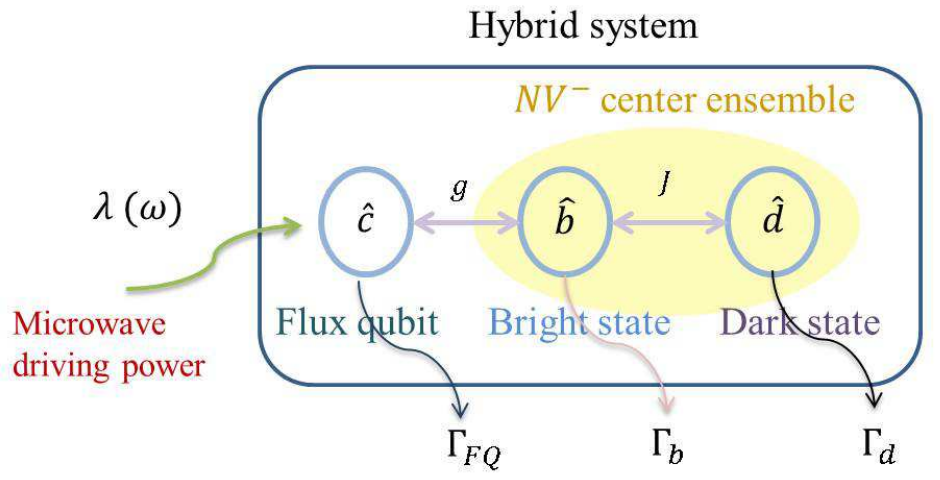

Figure 10. Schematic of the model for the THOM to describe superconductor diamond hybrid system. The flux qubit is considered as a harmonic operator. The bright state is coupled with both the flux qubit and the dark state.

follows.

$$
\begin{aligned}
& H=\hbar \omega_{F Q} \hat{c}^{\dagger} \hat{c}+\hbar \omega_{N V} \hat{b}^{\dagger} \hat{b}+\hbar \omega_{N V} \hat{d}^{\dagger} \hat{d}+\hbar g\left(\hat{c}^{\dagger} \hat{b}+\hat{c} \hat{b}^{\dagger}\right) \\
& +\hbar J\left(e^{i \theta} \hat{b}^{\dagger} \hat{d}+e^{-i \theta} \hat{b} \hat{d}^{\dagger}\right)+\lambda\left(\hat{c}^{\dagger}+\hat{c}\right) \cos (\omega t)-i \Gamma_{c} \hat{c}^{\dagger} \hat{c}-i \Gamma_{b} \hat{b}^{\dagger} \hat{b}-i \Gamma_{d} \hat{d}^{\dagger} \hat{d}
\end{aligned}
$$

, which we call a three harmonic oscillator model (THOM). Here, $\lambda$ denotes the amplitude of the driving power and $\omega$ denotes the frequency of the driving power. $\Gamma$ denotes the energy relaxation term for flux qubit, bright state and dark state.

After performing the rotating wave approximation with the Hamiltonian described in Eq. (37), we have:

$$
\begin{aligned}
& H=\hbar \omega_{F Q}^{\prime} \hat{c}^{\dagger} \hat{c}+\hbar \omega_{N V}^{\prime} \hat{b}^{\dagger} \hat{b}+\hbar \omega_{N V}^{\prime} \hat{d}^{\dagger} \hat{d}+\hbar g\left(\hat{c}^{\dagger} \hat{b}+\hat{c} \hat{b}^{\dagger}\right)+\hbar J\left(e^{i \theta} \hat{b}^{\dagger} \hat{d}+e^{-i \theta} \hat{b} \hat{d}^{\dagger}\right) \\
& +\frac{1}{2} \lambda\left(\hat{c}^{\dagger}+\hat{c}\right)-i \Gamma_{c} \hat{c}^{\dagger} \hat{c}-i \Gamma_{b} \hat{b}^{\dagger} \hat{b}-i \Gamma_{d} \hat{d}^{\dagger} \hat{d}
\end{aligned}
$$

where $\omega_{F Q}^{\prime}=\omega_{F Q}-\omega$ denotes the difference between the flux qubit frequency and driving power frequency. $\omega_{N V}^{\prime}=\omega_{N V}-\omega$ denotes the frequency difference between $\mathrm{NV}^{-}$center and microwave driving power.

Based on this Hamiltonian, we obtain the following Heisenberg equation:

$$
\frac{d}{d t} \widehat{c}=-i[\hat{c}, H]=-i\left(\omega_{F Q}^{\prime} \hat{c}+g \hat{b}+\lambda-i \Gamma_{c} \hat{c}\right)
$$


a)

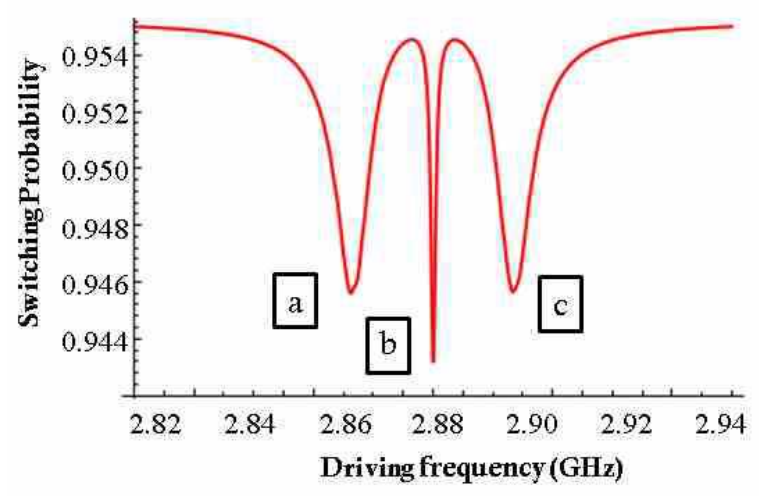

b)

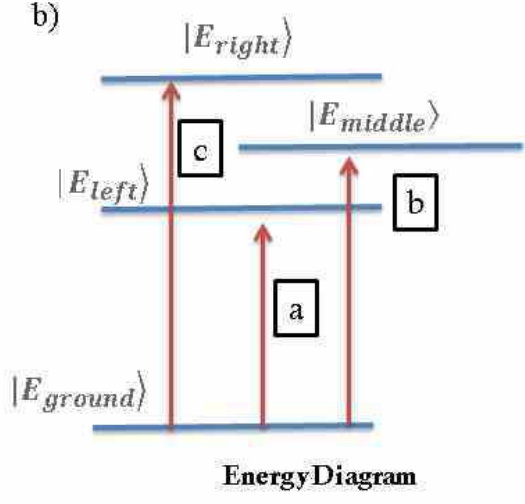

Figure 11. The relationship between the spectrum and energy level. In the picture (a), we plot the spectrum using the THOM where $\omega_{F Q} / 2 \pi=\omega_{N V} / 2 \pi=2.88 \mathrm{GHz}$, $g=13.0 \times 2 \pi \mathrm{MHz}, J=3.46 \times 2 \pi \mathrm{MHz}, \Gamma_{F Q}=0.30 \times 2 \pi \mathrm{MHz}, \Gamma_{d}=0.50 \times 2 \pi$ $\mathrm{MHz}, \Gamma_{b}=6.40 \times 2 \pi \mathrm{MHz}$, and $\lambda=1.00 \mathrm{MHz}$. The spectrum denotes the transition from a ground state to each excited state. In the picture (b), we describe the energy levels diagram. The transition between the ground state and the first (third) excited state $\left|E_{\text {left }}\right\rangle\left(\left|E_{\text {right }}\right\rangle\right)$ corresponds to the left (right) broad peak in the spectrum. The transition between the ground and the second excited state $\left|E_{\text {middle }}\right\rangle$ corresponds to the middle narrow peak.

$$
\begin{aligned}
& \frac{d}{d t} \widehat{b}=-i[\hat{b}, H]=-i\left(\omega_{N V}^{\prime} \hat{d}+g \hat{c}+J e^{i \theta} \hat{d}-i \Gamma_{b} \hat{b}\right) \\
& \frac{d}{d t} \widehat{d}=-i[\hat{d}, H]=-i\left(\omega_{N V}^{\prime} \hat{d}+J e^{-i \theta} \hat{d}-i \Gamma_{d} \hat{d}\right)
\end{aligned}
$$

We assume that, after driving the system for a long time, we obtain a steady state and so we obtain:

$$
\left(\frac{d}{d t} \hat{c}\right)_{t \rightarrow \infty}=\left(\frac{d}{d t} \hat{b}\right)_{t \rightarrow \infty}=\left(\frac{d}{d t} \hat{d}\right)_{t \rightarrow \infty}=0
$$

By solving these, the excited probability of the qubit is described as:

$$
\left\langle\hat{c}^{\dagger} \hat{c}\right\rangle=\left(\frac{\lambda}{2}\right)^{2} \times\left|\frac{\left[\left(i \Gamma_{b}-\omega_{b}^{\prime}\right)\left(i \Gamma_{d}-\omega_{d}^{\prime}\right)-J^{2}\right]}{\left(i \Gamma_{c}-\omega_{c}^{\prime}\right)\left[\left(i \Gamma_{b}-\omega_{b}^{\prime}\right)\left(i \Gamma_{d}-\omega_{d}^{\prime}\right)-J^{2}\right]-g^{2}\left(i \Gamma_{d}-\omega_{d}^{\prime}\right)}\right|^{2}(
$$

where $\omega_{F Q}^{\prime}=\omega_{F Q}-\omega$ and $\omega_{N V}^{\prime}=\omega_{N V}-\omega$. We plot this in Fig. 11, and this can reproduce three peaks that are observed in this hybrid system [25].

From the THOM, we confirm that the phase $\theta$ in the expression of coupling between the dark state and the bright state would not affect the spectroscopic measurement results, and therefore, we set $\theta=0$ throughout this paper.

5.5.2. Properties of the THOM with a resonant condition We investigate the behavior of this THOM for several parameters when the flux qubit is resonant with the $\mathrm{NV}^{-}$ centers. From the simulation result, the hybrid system contains three excited states, and the middle peak in the spectroscopy denotes the hybrid dark state whose width is much narrower than that of two side peaks. In order to understand the effect of each 
a)

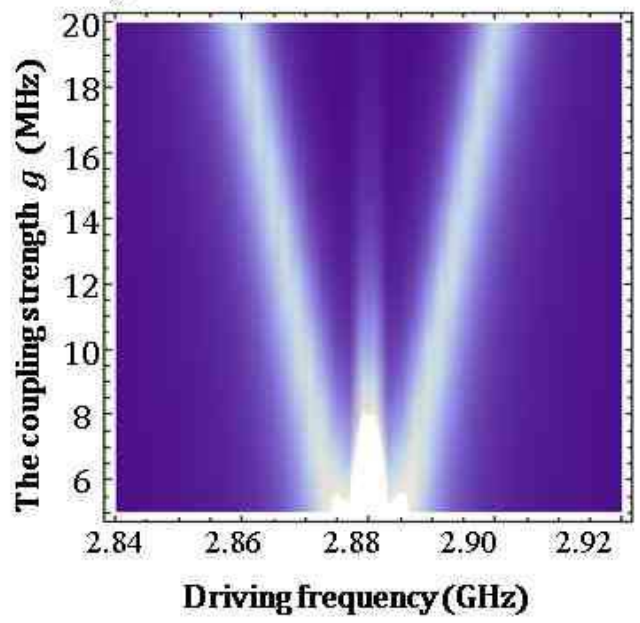

b)

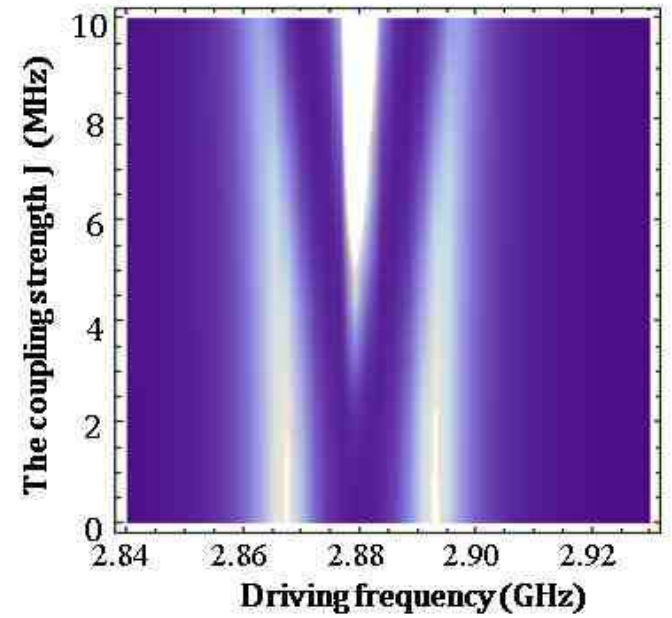

Figure 12. Density plot of the spectroscopy of the THOM to describe the dependency on $J$ and $g$ where $\Gamma_{F Q}=0.30 \times 2 \pi \mathrm{MHz}, \Gamma_{d}=0.50 \times 2 \pi \mathrm{MHz}, \Gamma_{b}=6.40 \times 2 \pi \mathrm{MHz}$, and $\lambda=1 \times 2 \pi \mathrm{MHz}$. In the picture (a), as the value of $g$ increases, the middle peak becomes darker. Meanwhile, the two side peaks will be largely split for a larger $g$. On the other hand, in the picture (b), as the value of $J$ increases, the effective coupling between the dark state and the flux qubit becomes stronger where the bright state mediates the interaction, which makes the middle peak higher than the other two peaks.

parameter, we draw a density plot and show the relationship between the parameters and spectroscopy (shown in Fig,12 and Fig 13).

As the value of $g$ increases, the separation between two peaks becomes larger (shown in Fig 12 a). This can be explained by an increase of the vacuum Rabi splitting that is determined by the $J$ and $g$ as follows.

$$
\Delta E_{\text {right }}-\Delta E_{\text {left }}=2 \hbar \sqrt{g^{2}+J^{2}}
$$

where the value of $g$ depends on both a persistent current of the flux qubit and the distance between $\mathrm{NV}^{-}$center and flux qubit. The value of $J$ is determined by the inhomogeneous effect of $\mathrm{NV}^{-}$centers. Since $g$ is usually much larger than $J$, we have $\Delta E_{\text {right }}-\Delta E_{\text {left }} \simeq 2 \hbar\left(g+\frac{J^{2}}{g}\right.$ ) (here, $g \gg J$ ) and so this energy gap is mainly determined by the value of $g$. The area of the peak is determined by the form of the eigenvectors. From the expression of $\left|E_{\text {middle }}\right\rangle$, as $g$ increases, the weight of $|D \downarrow\rangle$ increases while the weight of the flux qubit $|0 \uparrow\rangle$ decreases. This means that, for a large $g$, it becomes more difficult to detect the dark state by the spectroscopy where only the flux qubit excitation is measured, and the middle peak disappears (See the Fig 12 a).

As the value of $J$ increases, the coupling between the dark state and bright state becomes stronger, which induces a stronger hybridization between dark state and flux qubit. Since the weight of $|0 \uparrow\rangle$ increases with enlarging $J$, the area of the middle peak becomes larger (See the Fig $12 \mathrm{~b}$ ).

If we change the decay rate $\Gamma_{b}$ of the bright state, it will make two sides peaks 
a)

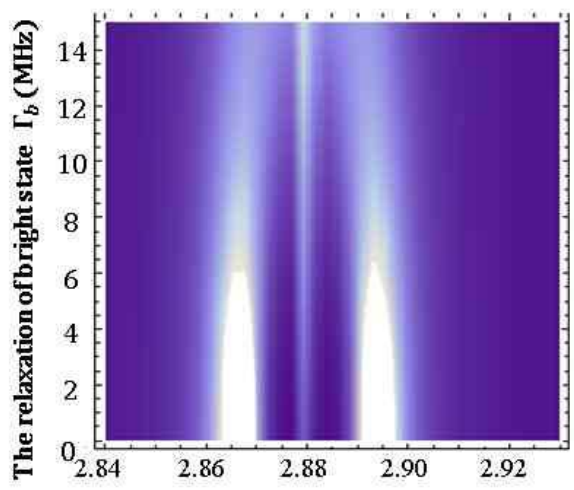

c)

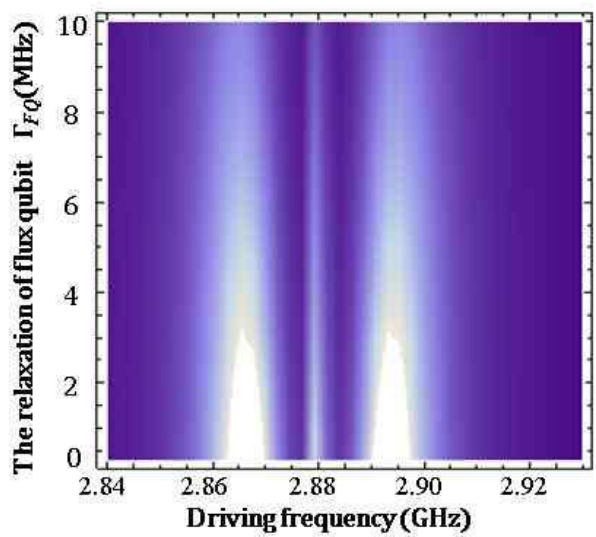

b)

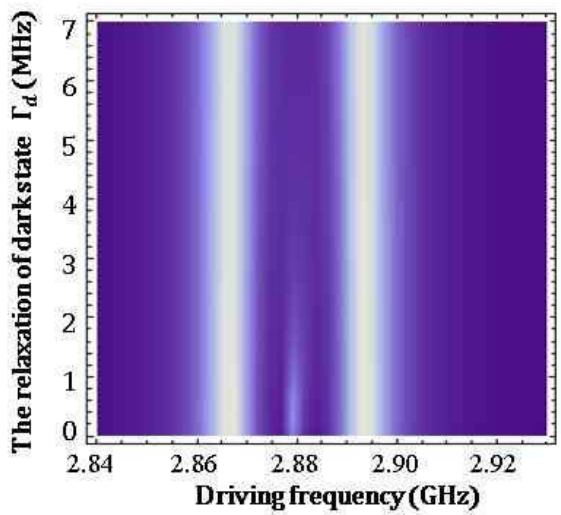

Figure 13. Density plot of the spectroscopy to describe the dependency on the decay rate $\Gamma_{b}, \Gamma_{d}$, and $\Gamma_{F Q}$, respectively. We fix the other parameters $g=13.0 \times 2 \pi \mathrm{MHz}$; $J=3.46 \times 2 \pi \mathrm{MHz} ; \lambda=1 \times 2 \pi \mathrm{MHz}$ and then change the $\Gamma_{b}, \Gamma_{d}$, and $\Gamma_{F Q}$ values. These three pictures (a), (b) and (c) correspond to the excited population varieties with $\Gamma_{b}, \Gamma_{d}$, and $\Gamma_{F Q}$. In the picture (a), $\Gamma_{b}$ mainly affects the width of the side peak which becomes broader as the value of $\Gamma_{b}$ increases. On the other hand, the width of the middle peak is insensitive against $\Gamma_{b}$. In the picture (b), the relaxation rate of the dark sate $\Gamma_{d}$ has a significant contribution to the excited population of middle peak but has a smaller influence on the side peaks. The picture (c) shows the effect of $\Gamma_{F Q}$, and it change the width of the side peaks and has insignificant effect on the middle peak. The side peaks decay rapidly as $\Gamma_{F Q}$ increase.

broader (shown in Fig 13 a), due to a shorter life time. For the effect of $\Gamma_{d}$, it will only contribute to the middle peak and has almost no influence on the side peaks (shown in Fig $13 \mathrm{~b}$ ), which is consistent with the form of the eigenvector described in Eq 32 and Eq $36(J \ll g)$. The flux qubit is directly coupled with bright state and is coupled with the dark state indirectly. Therefore, the flux qubit decay rate $\Gamma_{F Q}$ mainly affects the width of the side peaks, while it has smaller effect on the middle peak (shown in Fig.13 c).

5.5.3. Properties of the THOM with a detuning Next we consider a case to add the detuning between the flux qubit and $\mathrm{NV}^{-}$center. We define the detuning as $\delta=\omega_{F Q}-\omega_{N V}$. As long as the detuning is much smaller than other parameters, it is valid to regard the detuning as the perturbation to the system. We rewrite the 
a)

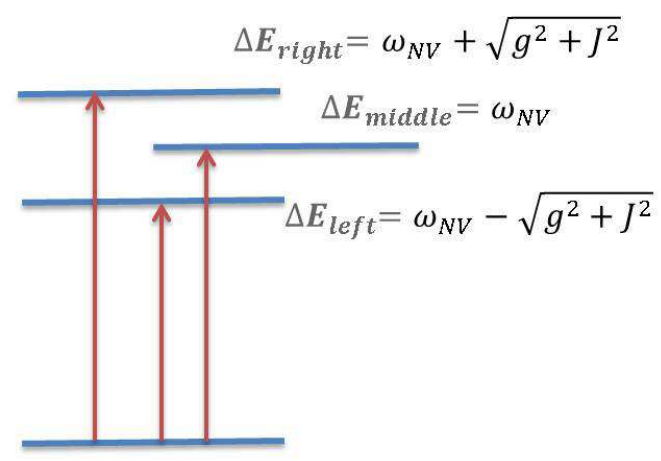

b)

$$
\Delta E_{\text {right }}=\omega_{N V}+\sqrt{g^{2}+J^{2}}+\frac{1}{2} \delta \frac{g^{2}}{g^{2}+J^{2}}
$$

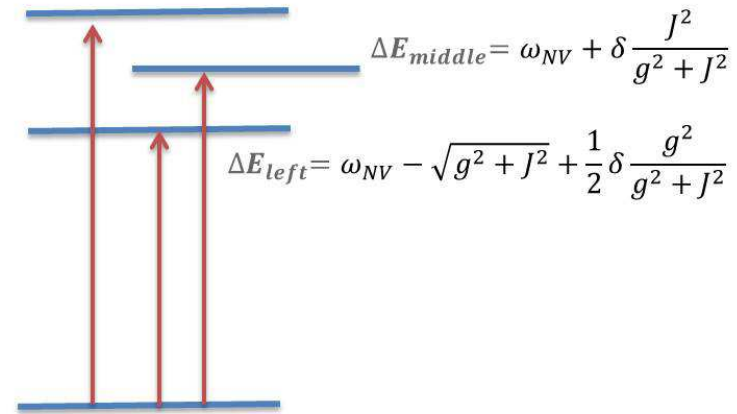

Figure 14. The energy level diagram with and without detuning. Picture (a) shows the energy level and energy gap between ground state and each excited state without detuning. Picture (b) shows the effect of inducing the detuning between flux qubit and $\mathrm{NV}^{-}$center. From the energy level diagram, the frequency of each peak will cause a blue shift depending on the $\delta$ value.

Hamiltonian as following:

$$
H=\hbar\left(\omega_{N V}+\delta\right) \hat{c}^{\dagger} \hat{c}+\hbar \omega_{N V} \hat{b}^{\dagger} \hat{b}+\hbar \omega_{N V} \hat{d}^{\dagger} \hat{d}+\hbar g\left(\hat{c}^{\dagger} \hat{b}+\hat{c} \hat{b}^{\dagger}\right)+\hbar J\left(\hat{b}^{\dagger} \hat{d}+\hat{b} \hat{d}^{\dagger}\right)
$$

By diagonalising the Hamiltonian and using the perturbation theory, we can calculate the eigenvalues and eigenvectors (shown in Fig.14).

$$
\begin{aligned}
& \Delta E_{\text {left }}=E_{\text {left }}-E_{0} \approx \hbar\left(\omega_{N V}-\sqrt{g^{2}+J^{2}}+\frac{1}{2} \delta \frac{g^{2}}{g^{2}+J^{2}}\right) \\
& \left|E_{\text {left }}\right\rangle=-\frac{1}{\sqrt{2}}\left[1+\frac{g^{2} \delta}{4\left(g^{2}+J^{2}\right)^{3 / 2}}\right] \cdot|B \downarrow\rangle \\
& +\frac{1}{\sqrt{2}} \frac{g}{\sqrt{g^{2}+J^{2}}}\left[1-\frac{g^{2} \delta}{4\left(g^{2}+J^{2}\right)^{3 / 2}}-\frac{J^{2} \delta}{\left(g^{2}+J^{2}\right)^{3 / 2}}\right] \cdot|0 \uparrow\rangle \\
& +\frac{1}{\sqrt{2}} \frac{J}{\sqrt{g^{2}+J^{2}}}\left[1-\frac{g^{2} \delta}{4\left(g^{2}+J^{2}\right)^{3 / 2}}+\frac{J^{2} \delta}{\left(g^{2}+J^{2}\right)^{3 / 2}}\right] \cdot|D \downarrow\rangle \\
& \Delta E_{\text {middle }}=E_{\text {middle }}-E_{0} \approx \hbar\left(\omega_{N V}+\delta \frac{J^{2}}{g^{2}+J^{2}}\right) \\
& \left|E_{\text {middle }}\right\rangle=-\frac{J}{\sqrt{g^{2}+J^{2}}}|0 \uparrow\rangle+\frac{g}{\sqrt{g^{2}+J^{2}}}|D \downarrow\rangle+\delta \frac{g J}{\left(g^{2}+J^{2}\right)^{3 / 2}}|B \downarrow\rangle \\
& \Delta E_{\text {right }}=E_{\text {right }}-E_{0} \approx \hbar\left(\omega_{N V}+\sqrt{g^{2}+J^{2}}+\frac{1}{2} \delta \frac{g^{2}}{g^{2}+J^{2}}\right) \\
& \left|E_{\text {right }}\right\rangle=-\frac{1}{\sqrt{2}}\left[1-\frac{g^{2} \delta}{4\left(g^{2}+J^{2}\right)^{3 / 2}}\right] \cdot|B \downarrow\rangle
\end{aligned}
$$


a)
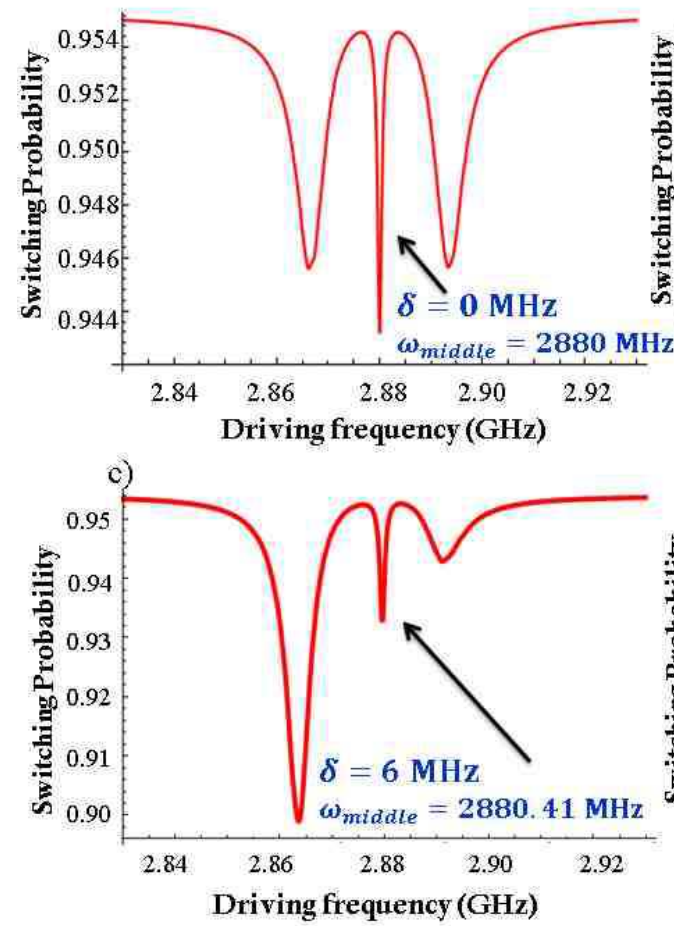

b)
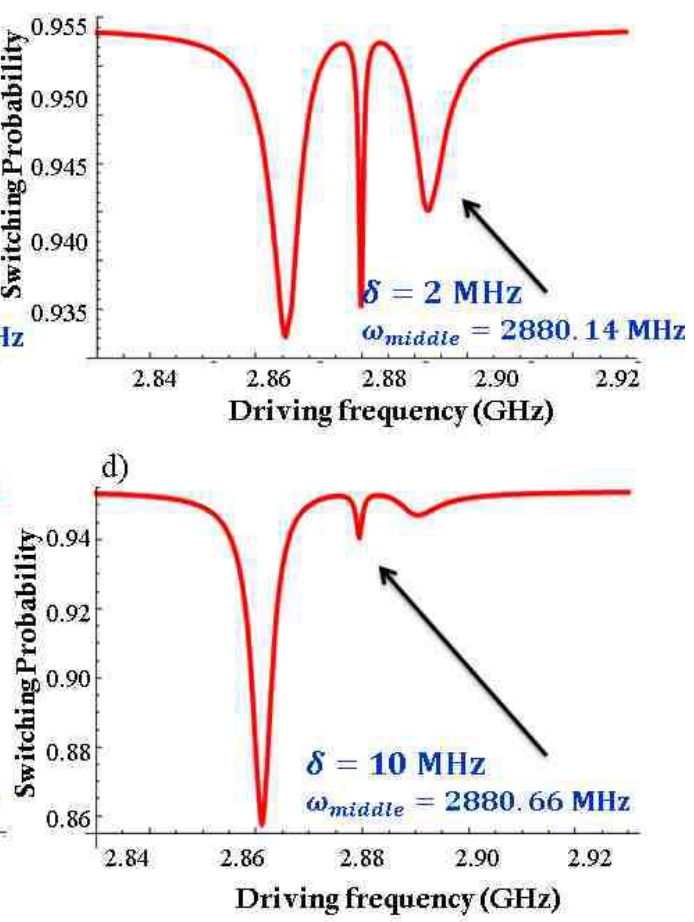

Figure 15. Energy shift of the middle peak against detuning for MHOM. Each picture presents for the case of the detuning as $0,2 \mathrm{MHz}, 6 \mathrm{MHz}$ and $10 \mathrm{MHz}$, respectively. The detuning makes it difficult to excite the left and middle peak whose frequency is far from the energy of the flux qubit. Here we choose the same parameters as in Fig 8 except of the frequency of the flux qubit which is equal to the sum of the detuning and frequency of $\mathrm{NV}^{-}$center.

a)

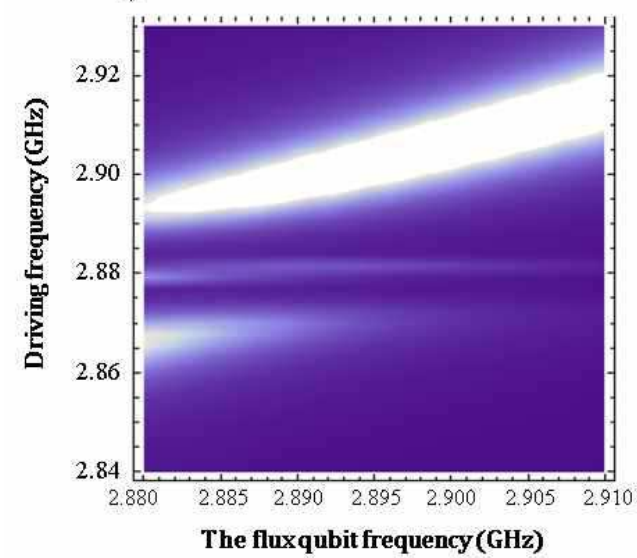

b)

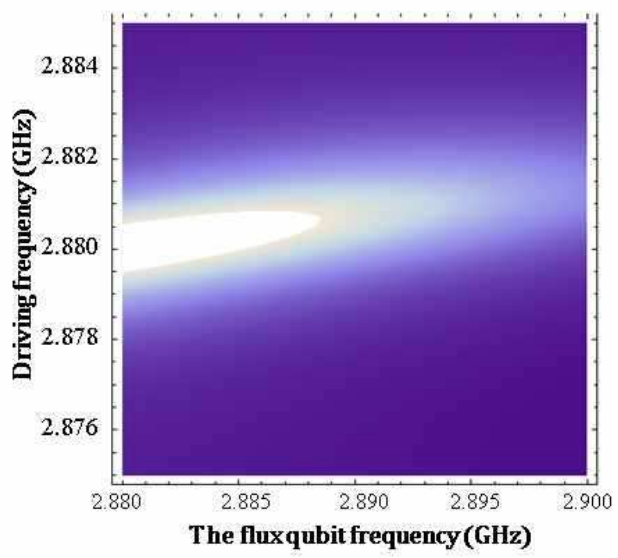

Figure 16. Numerical simulation by solving THOM where the $\mathrm{x}$ axis denotes the flux-qubit frequency and y axis denotes the microwave frequency. (a) Density plot of the spectroscopy. (b) Magnified view of the spectroscopy around 2.88 GHz. These pictures clearly show that every peak has a finite energy shift if we add the detuning. Here, we use the same parameters as in Fig. 11 


$$
\begin{aligned}
& +\frac{1}{\sqrt{2}} \frac{g}{\sqrt{g^{2}+J^{2}}}\left[1+\frac{g^{2} \delta}{4\left(g^{2}+J^{2}\right)^{3 / 2}}+\frac{J^{2} \delta}{\left(g^{2}+J^{2}\right)^{3 / 2}}\right] \cdot|0 \uparrow\rangle \\
& +\frac{1}{\sqrt{2}} \frac{J}{\sqrt{g^{2}+J^{2}}}\left[1+\frac{g^{2} \delta}{4\left(g^{2}+J^{2}\right)^{3 / 2}}-\frac{J^{2} \delta}{\left(g^{2}+J^{2}\right)^{3 / 2}}\right] \cdot|D \downarrow\rangle
\end{aligned}
$$

These results show that, as the detuning increases, the energy difference between the excited states and the ground state would increase. The amount of change depends on $g, J$, and $\delta$ (shown in Fig.15). It is worth mentioning that the middle peaks has a smaller energy shift than that of the other two side peaks for $g \gg J$.

From the spectroscopy calculated by MHOM, we have also confirmed the fact that the frequency of middle peak can be shifted by the detuning (See Fig.15 and Fig[16). Also, the left and right peaks have an energy shift for the detuning, and the energy shift is much larger than that of the middle peak. ( See the Fig,15 and Fig,16])

\section{References}

[1] J.-M. Pirkkalainen, S. U. Cho, Jian Li, G. S. Paraoanu, P. J. Hakonen and M. A. Sillanpää, Hybrid circuit cavity quantum electrodynamics with a micromechanical resonator, Nature 494, 211-215 (2013).

[2] Mucke, M., Figueroa, E., Bochmann, J., Hahn, C., Murr, K., Ritter, S., Villas-Boas, C. J., and Rempe, G. Electromagnetically induced transparency with single atoms in a cavity, Nature 465, 755-758 (2010).

[3] Sørensen, Anders S and van der Wal, Caspar H and Childress, Lilian I and Lukin, Mikhail D, Capacitive coupling of atomic systems to mesoscopic conductors. Phys. Rev. Lett. 92, 063601 (2004).

[4] Tian, L and Rabl, P and Blatt, R and Zoller, P., Interfacing quantum-optical and solid-state qubits. Phys. Rev. Lett. 92, 247902 (2004).

[5] A. Wallraff, D. I. Schuster, A. Blais, L. Frunzio, R.-S. Huang, J. Majer, S. Kumar, S. M. Girvin and R. J. Schoelkopf, Circuit quantum electrodynamics: Coherent coupling of a single photon to a Cooper pair box, Nature 431, 162 (2004).

[6] Rabl, P and DeMille, D and Doyle, JM and Lukin, MD and Schoelkopf, RJ and Zoller, P., \& Sorensen, A. S. Hybrid quantum processors: molecular ensembles as quantum memory for solid state circuits. Phys. Rev. Lett. 97, 033003 (2006).

[7] Marcos, D., Wubs, M., Taylor, J. M., Aguado, R., Lukin, M. D., \& Sorensen, A. S. Coupling Nitrogen-Vacancy Centers in Diamond to Superconducting Flux Qubits. Phys. Rev. Lett. 105, 210501 (2010).

[8] T. D. Ladd, P. van Loock, K. Nemoto, W. J. Munro, and Y Yamamoto, Hybrid quantum repeater based on dispersive CQED interactions between matter qubits and bright coherent light. New Journal of Physics 8, 184 (2006).

[9] Clarke, John and Wilhelm, Frank K Superconducting quantum bits, Nature 453, 1031-1042 (2008).

[10] Nakamura, Yu and Pashkin, Yu A and Tsai, JS Coherent control of macroscopic quantum states in a single-Cooper-pair box, Nature 398, 786-788 (1999).

[11] Pirkkalainen, Vion, D and Aassime, A and Cottet, A and Joyez, Pl and Pothier, H and Urbina, C and Esteve, D and Devoret, Michel H Manipulating the quantum state of an electrical circuit, Science 296, 886-889 (2002).

[12] Chiorescu, I and Nakamura, Y and Harmans, CJP Ma and Mooij, JE Coherent quantum dynamics of a superconducting flux qubit, Science 299, 1869-1871 (2003).

[13] Sillanpää, Mika A and Park, Jae I and Simmonds, Raymond W Coherent quantum state storage and transfer between two phase qubits via a resonant cavity, Nature 449, 438-442 (2007). 
[14] Majer, J and Chow, JM and Gambetta, JM and Koch, Jens and Johnson, BR and Schreier, JA and Frunzio, L and Schuster, DI and Houck, AA and Wallraff, Andreas and others Coupling superconducting qubits via a cavity bus, Nature 449, 443-447 (2007).

[15] DiCarlo, L and Chow, JM and Gambetta, JM and Bishop, Lev S and Johnson, BR and Schuster, DI and Majer, J and Blais, A and Frunzio, L and Girvin, SM and others Demonstration of two-qubit algorithms with a superconducting quantum processor, Nature 460, 240-244 (2009).

[16] Ansmann, Markus and Wang, $\mathrm{H}$ and Bialczak, Radoslaw $\mathrm{C}$ and Hofheinz, Max and Lucero, Erik and Neeley, M and O'Connell, AD and Sank, D and Weides, M and Wenner, J and others Violation of Bell's inequality in Josephson phase qubits, Nature 461, 504-506 (2009).

[17] R. Barends, J. Kelly, A. Megrant, A. Veitia, D. Sank, E. Jeffrey, T. C. White, J. Mutus, A. G. Fowler, B. Campbell, Y. Chen, Z. Chen, B. Chiaro, A. Dunsworth, C. Neill, P. Malley, P. Roushan, A. Vainsencher, J. Wenner, A. N. Korotkov, A. N. Cleland and John M. Martinis, Superconducting quantum circuits at the surface code threshold for fault tolerance, Nature $\mathbf{5 0 8}$, 500-503 (2014).

[18] Imamoglu, A. Cavity QED based on collective magnetic dipole coupling: spin ensembles as hybrid two-level systems, Phys. Rev. Lett. 102, 083602 (2009).

[19] Wesenberg, J. H., Ardavan, A., Briggs, G. A. D., Morton, J. J. L., Schoelkopf, R. J. , Schuster, D.I., \& MolmerK. Quantum Computing with an Electron Spin Ensemble, Phys. Rev. Lett. 103, 070502 (2009).

[20] Kubo, Yuimaru and Grezes, Cecile and Dewes, Andreas and Umeda, T and Isoya, Junichi and Sumiya, H and Morishita, N and Abe, H and Onoda, S and Ohshima, T and others, Hybrid quantum circuit with a superconducting qubit coupled to a spin ensemble. Phys. Rev. Lett. 107, 220501 (2011).

[21] Kubo, Y., Ong, F.R., Bertet, P., Vion, D. , Jacques, V., Zheng, D., Dreau, A., Roch, J. F., Auffeves, A., Jelezko, F., Wrachtrup, J., Barthe, M. F., Bergonzo, P., \& Esteve, D. Strong coupling of a spin ensemble to a superconducting resonator, Phys. Rev. Lett. 105, 140502 (2010).

[22] Kubo, Y., Diniz, I., Dewes, A., Jacques, V., Dréau, A., Roch, J.F., Auffeves, A., Vion, D., Esteve, D. and Bertet, P. Storage and retrieval of a microwave field in a spin ensemble, Phys. Rev. A 85, 012333 (2012).

[23] Zhu, Xiaobo and Kemp, Alexander and Saito, Shiro and Semba, Kouichi Coherent operation of a gap-tunable flux qubit, Applied Physics Letters 97, 102503 (2010).

[24] Saito, S., Zhu, X., Amsuss, R., Matsuzaki, Y., Kakuyanagi, K., Shimo-Oka, T., Mizuochi, N., Nemoto, K., Munro, W. J., \& Semba, K. Towards realizing a quantum memory for a superconducting qubit: Storage and retrieval of quantum states, Phys. Rev. Lett 111, 107008 (2013).

[25] Zhu, Xiaobo and Matsuzaki, Yuichiro and Amsuss, Robert and Kakuyanagi, Kosuke and ShimoOka, Takaaki and Mizuochi, Norikazu and Nemoto, Kae and Munro, William J and Semba, Kouichi and Saito, Shiro Observation of dark states in a superconductor diamond quantum hybrid system, Nature communications 3424, 4524 (2014).

[26] Matsuzaki, Yuichiro and Zhu, Xiaobo and Kakuyanagi, Kosuke and Shimo-Oka, Takaaki and Mizuochi, Norikazu and Nemoto, Kae and Semba, Kouichi and Munro, William J and Yamaguchi, Hiroshi and Saito, Shiro Improving the lifetime of the NV center ensemble coupled with a superconducting flux qubit by applying magnetic fields, Rev. A. 91, 4, 042329 (2015)

[27] Mizuochi, N and Neumann, P and Rempp, F and Beck, J and Jacques, V and Siyushev, P and Nakamura, K and Twitchen, DJ and Watanabe, H and Yamasaki, S and others, Coherence of single spins coupled to a nuclear spin bath of varying density, Phys. Rev. B 80, 041201 (2009).

[28] Balasubramanian, Gopalakrishnan and Neumann, Philipp and Twitchen, Daniel and Markham, Matthew and Kolesov, Roman and Mizuochi, Norikazu and Isoya, Junichi and Achard, Jocelyn and Beck, Johannes and Tissler, Julia and others Ultralong spin coherence time in isotopically engineered diamond, Nature materials 8, Nature materials (2009).

[29] Y. Kubo, I. Diniz, C. Grezes, T. Umeda, J. Isoya, H. Sumiya, T. Yamamoto, H. Abe, S. Onoda, 
T. Ohshima, V. Jacques, A. Dréau, J.-F. Roch, A. Auffeves, D. Vion, D. Esteve, and P. Bertet, Hybrid quantum circuit with a superconducting qubit coupled to a spin ensemble. Phys. Rev. $B$ 86, 064514 (2012).

[30] Amsüss, Robert and Koller, Ch and Nöbauer, Tobias and Putz, Stefan and Rotter, Stefan and Sandner, Kathrin and Schneider, Stephan and Schramböck, Matthias and Steinhauser, Georg and Ritsch, Helmut and others, Cavity QED with magnetically coupled collective spin states. Phys. Rev. Lett. 107, 060502 (2011).

[31] Schuster, DI and Sears, AP and Ginossar, E and DiCarlo, L and Frunzio, L and Morton, JJL and $\mathrm{Wu}, \mathrm{H}$ and Briggs, GAD and Buckley, BB and Awschalom, DD and others, High-cooperativity coupling of electron-spin ensembles to superconducting cavities. Phys. Rev. Lett. 105, 140501 (2010).

[32] S. Putz, D. O. Krimer, R. Amsüss, A. Valookaran, T. Nöbauer, J. Schmiedmayer, S. Rotter and J. Majer, Protecting a spin ensemble against decoherence in the strong-coupling regime of cavity QED. NATURE PHYSICS 10, 720-724 (2014).

[33] F. G. Paauw, A. Fedorov, C. J. P. M Harmans, and J. E. Mooij, Tuning the gap of a superconducting flux qubit. Phys. Rev. Lett. 102, 090501 (2009).

[34] Zhu, Xiaobo and Saito, Shiro and Kemp, Alexander and Kakuyanagi, Kosuke and Karimoto, Shinichi and Nakano, Hayato and Munro, William J and Tokura, Yasuhiro and Everitt, Mark S and Nemoto, Kae and others, Coherent coupling of a superconducting flux qubit to an electron spin ensemble in diamond. Nature 478, 221-224 (2011).

[35] Diniz, I., Portolan, S., Ferreira, R., Gerard, J. M., Bertet, P., \& Auffeves, A. Strongly coupling a cavity to inhomogeneous ensembles of emitters: Potential for long-lived solid-state quantum memories, Phys. Rev. A 84, 063810 (2011).

[36] M. Fleischhauer and M. D. Lukin, Quantum memory for photons: Dark-state polaritons, Phys. Rev. A 65, 022314 (2002).

[37] Ford, GW and O'Connell, RF, Comment on Dissipative Quantum Dynamics with a Lindblad Functional, Phys. Rev. Lett. 82, 3376-3376 (1999).

[38] Prosen, Tomaž, Spectral theorem for the Lindblad equation for quadratic open fermionic systems, Journal of Statistical Mechanics: Theory and Experiment 2010, P07020 (2010).

[39] Ishizaki, Akihito and Fleming, Graham R, On the adequacy of the Redfield equation and related approaches to the study of quantum dynamics in electronic energy transfer, The Journal of chemical physics 130, 234110 (2009). 